\title{
Entanglement dynamics for Unruh-DeWitt detectors interacting with massive scalar fields: the Unruh and anti-Unruh effects
}

\author{
Yuebing Zhou, ${ }^{a, b}$ Jiawei $\mathbf{H u}^{a, 1}$ and Hongwei $\mathbf{Y u}^{a, 2}$ \\ ${ }^{a}$ Department of Physics and Synergetic Innovation Center for Quantum Effects and Applications, \\ Hunan Normal University, \\ 36 Lushan Rd., Changsha, Hunan 410081, China \\ ${ }^{b}$ Department of Physics, Huaihua University, \\ 180 Huaidong Rd., Huaihua, Hunan 418008, China \\ E-mail: 258286519@qq.com, jwhu@hunnu.edu.cn, hwyu@hunnu.edu.cn
}

ABSTRACT: We study, in the framework of open quantum systems, the entanglement dynamics for a quantum system composed of two uniformly accelerated Unruh-Dewitt detectors interacting with a bath of massive scalar fields in the Minkowski vacuum. We find that the entanglement evolution for the quantum system coupled with massive fields is always slower compared with that of the one coupled with massless fields, and this time-delay effect brought about by the field being massive can however be counteracted by a large enough acceleration, in contrast to the case of a static quantum system in a thermal bath, where this time delay is not affected by the temperature. Remarkably, the maximal concurrence of the quantum system generated during evolution may increase with acceleration for any inter-detector separation while that for static ones in a thermal bath decreases monotonically with temperature, and this can be considered as an anti-Unruh effect in terms of the entanglement generated.

KEYwords: Models of Quantum Gravity, Quantum Dissipative Systems, Thermal Field Theory

ArXIV EPRINT: 2105.14735

\footnotetext{
${ }^{1}$ Corresponding author.

${ }^{2}$ Corresponding author.
} 


\section{Contents}

1 Introduction $\quad 2$

2 The basic formalism 3

3 Entanglement dynamics of a uniformly accelerated quantum system $\begin{array}{lr}\text { coupled with massive scalar fields } & 9\end{array}$

3.1 The time-delay effect $\quad 9$

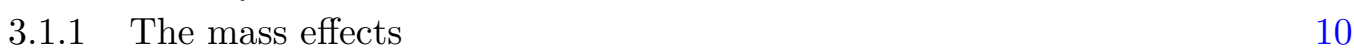

$\begin{array}{lll}3.1 .2 & \text { The acceleration effects } & 10\end{array}$

$\begin{array}{ll}3.2 & \text { The entanglement degradation effect } \\ \end{array}$

$\begin{array}{lll}3.2 .1 & \text { Entanglement degradation caused by dissipation } & 12\end{array}$

$\begin{array}{ll}\text { 3.2.2 Entanglement degradation caused by decoherence } & 13\end{array}$

$\begin{array}{ll}3.3 & \text { The entanglement generation effect } \\ \end{array}$

$\begin{array}{lll}\text { 3.3.1 The mass effects } & 17\end{array}$

$\begin{array}{lll}\text { 3.3.2 The acceleration effects } & 18\end{array}$

3.4 The Unruh and anti-Unruh effects 20

$\begin{array}{lll}4 & \text { Summary } & 22\end{array}$

A The expressions of the entanglement dynamic parameters in various cases

A.1 The acceleration case 24

A.2 The thermal bath case 24

B The limit properties of $\Omega_{a}(m / \omega, a / \omega) \quad \mathbf{2 5}$

B.1 The low-mass limit 25

B.2 The high-mass limit 25

B.3 The low-acceleration limit 26

B.4 The high-acceleration limit 26

C The limit properties of $\lambda_{a}(m / \omega, a / \omega, L \omega) \quad 26$

$\begin{array}{ll}\text { C.1 The low-mass limit } & 27\end{array}$

$\begin{array}{ll}\text { C.2 The high-mass limit } & 27\end{array}$

C.3 The low-acceleration limit $\quad 27$

$\begin{array}{ll}\text { C.4 The high-acceleration limit } & 27\end{array}$

D The limit properties of $\zeta_{a}^{ \pm}(m / \omega, a / \omega, L \omega) \quad 28$

$\begin{array}{lll}\text { D.1 The low-mass limit } & 28\end{array}$

D.2 The high-mass limit 28

D.3 The low-acceleration limit 28

D.4 The high-acceleration limit 29 


\section{Introduction}

Quantum field theory predicts that a uniformly accelerated observer perceives the vacuum of an inertial observer as a thermal bath at a temperature proportional to its proper acceleration, which is known as the Unruh effect [1-4]. A widely used model of uniformly accelerated observers is the Unruh-DeWitt detector, which is typically modeled as a point-like two-level quantum system coupled with fluctuating vacuum quantum fields [3, 5]. In fact, the thermal bath perceived by the uniformly accelerated observer can be considered as the Rindler thermal bath, which may not necessarily be same as the Minkowski thermal bath seen by an inertial observer. Many studies have been committed to comparing the different behaviors between quantum systems immersed in these two kinds of thermal baths from different physical aspects, e.g. the spontaneous emission rates [6-9], the Lamb shifts [10, 11], the resonance interactions $[12,13]$ and the Casimir-Polder interactions [14-16]. It has been shown that, only in some special situations, the behaviors of a single Unruh-DeWitt detector in the two kinds of thermal baths are equivalent, such as in the case of massless scalar fields in a free spacetime $[7,10]$.

Recently, the influences of environment on the entanglement dynamics of an open quantum system have been extensively studied, such as those leading to the environmentinduced entanglement sudden death [17, 18], entanglement revival [19] and entanglement creation [20-32]. Therefore, a question naturally arises as to how the entanglement dynamics of a quantum system composed of two Unruh-DeWitt detectors will be affected by acceleration, and how will it be different from that of a static one in a thermal bath in the Minkowski spacetime at the Unruh temperature related to acceleration. In ref. [33], the entanglement generation of two uniformly accelerated Unruh-DeWitt detectors coupled with fluctuating massless scalar fields in the Minkowski vacuum with a vanishing separation has been studied, and it has been shown that the asymptotic entanglement is exactly the same as that immersed in a thermal bath at the Unruh temperature. However, in more general cases, e.g. when the separation between the detectors is nonzero [34-36], in the presence of a boundary [36, 37], and for the quantum systems coupled with different kinds of quantum fields, such as electromagnetic fields [35, 36], the differences between the entanglement dynamics of an accelerated quantum system and that of a static one in a thermal bath show up.

When an Unruh-DeWitt detector is coupled with massive scalar fields, transitions among different eigenstates for an accelerated detector can still occur even when the mass of the field is greater than the energy level spacing of the detector $[4,6,8,38]$, which is impossible for a static one in a thermal bath. Furthermore, it has been shown in ref. [38] that the mass of the field will bring a gray factor related to acceleration to the transition rate of an Unruh-DeWitt detector, which results in the fact that the transition rate may decrease with acceleration. Later, this phenomenon is named as the anti-Unruh effect $[39,40]$. Recently, the entanglement dynamics of two static detectors coupled with massive scalar fields has been studied in ref. [41]. It has been found that, compared with the massless field case, the evolution of entanglement is slower and the region of spatial separation between the detectors within which entanglement can be generated is significantly 
enlarged. This means that it is possible to achieve long-distance entanglement generation and long-lived entanglement. Now, a natural question is, will there be essential differences between the behaviors of two uniformly accelerated Unruh-DeWitt detectors coupled with massive fields in the Minkowski vacuum and that of a static one in a thermal bath in terms of entanglement dynamics? In particular, will there be anti-Unruh phenomena, e.g. the entanglement generated for accelerated detectors increases with acceleration, while that for static ones in a thermal bath decreases with temperature? In the present paper, we study, in the framework of open quantum systems, the entanglement dynamics for a uniformly accelerated quantum system composed of two Unruh-DeWitt detectors interacting with a bath of fluctutating massive scalar fields in the Minkowski vacuum. Hereafter natural units with $\hbar=c=k_{B}=1$ are used unless specified, where $c$ is the speed of light, $\hbar$ the reduced Planck constant, and $k_{B}$ the Boltzmann constant.

\section{The basic formalism}

We consider a quantum system composed of a pair of Unruh-DeWitt detectors in interaction with a bath of fluctuating massive scalar fields in the Minkowski vacuum. The Hamiltonian of the total system takes the form

$$
H=H_{S}+H_{F}+H_{I}
$$

Here $H_{S}$ denotes the Hamiltonian of the quantum system, which can be generically written as

$$
H_{S}=\frac{\omega}{2} R_{3}^{(1)}+\frac{\omega}{2} R_{3}^{(2)},
$$

where $\omega$ is the energy level spacing between the excited state $|1\rangle$ and the ground state $|0\rangle$ of the Unruh-Dewitt detector, and $R_{\mu}^{(1)}=R_{\mu} \otimes R_{0}, R_{\mu}^{(2)}=R_{0} \otimes R_{\mu}$, with $R_{0}=|1\rangle\langle 1|+| 0\rangle\langle 0|$, $R_{1}=|0\rangle\langle 1|+| 1\rangle\langle 0|, R_{2}=i(|0\rangle\langle 1|-| 1\rangle\langle 0|)$, and $R_{3}=|1\rangle\langle 1|-| 0\rangle\langle 0| . H_{F}$ is the Hamiltonian of the massive scalar fields, the detail of which is not relevant here. The interaction Hamiltonian $H_{I}$ can be written in the following general form as [33]

$$
H_{I}=\varepsilon \sum_{\alpha=1}^{2} \sum_{\mu=0}^{3} R_{\mu}^{(\alpha)} \Phi_{\mu}\left(t_{\alpha}, \mathbf{x}_{\alpha}\right),
$$

where $\Phi_{\mu}(t, \mathbf{x})$ is the field operator, and $\varepsilon$ is the coupling constant which is assumed to be small. Now we assume that the scalar fields can be expanded as

$$
\Phi_{\mu}(x)=\sum_{k=1}^{N}\left[\chi_{\mu}^{k} \phi_{k}^{(-)}(x)+\left(\chi_{\mu}^{k}\right)^{*} \phi_{k}^{(+)}(x)\right]
$$

where $\phi_{k}^{( \pm)}(x)$ are positive and negative energy field operators relative to a set of $N$ independent massive scalar fields, and $\chi_{\mu}^{k}$ are the complex coefficients [33].

We assume that initially the quantum system is uncorrelated with the environment, i.e., the initial state of the total system can be written as $\rho_{\text {tot }}(0)=\rho(0) \otimes|0\rangle\langle 0|$, where $|0\rangle$ 
is the vacuum state of the massive scalar fields, and $\rho(0)$ denotes the initial state of the quantum system. The density matrix of the total system satisfies the Liouville equation

$$
\frac{\partial \rho_{\mathrm{tot}}(\tau)}{\partial \tau}=-i\left[H, \rho_{\mathrm{tot}}(\tau)\right]
$$

Under the Born-Markov approximation, the reduced density matrix of the quantum system $\rho(\tau)=\operatorname{Tr}_{F}\left[\rho_{\text {tot }}(\tau)\right]$ can be described by the Gorini-Kossakowski-Lindblad-Sudarshan (GKLS) master equation [42, 43],

$$
\frac{\partial \rho(\tau)}{\partial \tau}=-i\left[H_{\mathrm{eff}}, \rho(\tau)\right]+\mathcal{D}[\rho(\tau)]
$$

$H_{\text {eff }}$ and $\mathcal{D}[\rho(\tau)]$ in the equation above are the effective Hamiltonian and the dissipative term respectively, whose explicit form can be written as

$$
H_{\mathrm{eff}}=H_{S}-\frac{i}{2} \sum_{\alpha, \varrho=1}^{2}\left[H_{+}^{(\alpha \varrho)} R_{+}^{(\alpha)} R_{-}^{(\varrho)}+H_{-}^{(\alpha \varrho)} R_{-}^{(\alpha)} R_{+}^{(\varrho)}+H_{0}^{(\alpha \varrho)} R_{3}^{(\alpha)} R_{3}^{(\varrho)}\right],
$$

and

$$
\begin{aligned}
\mathcal{D}[\rho(\tau)]=\frac{1}{2} \sum_{\alpha, \varrho=1}^{2} & {\left[D_{+}^{(\alpha \varrho)}\left(2 R_{-}^{(\varrho)} \rho R_{+}^{(\alpha)}-\left\{R_{+}^{(\alpha)} R_{-}^{(\varrho)}, \rho\right\}\right)\right.} \\
+ & D_{-}^{(\alpha \varrho)}\left(2 R_{+}^{(\varrho)} \rho R_{-}^{(\alpha)}-\left\{R_{-}^{(\alpha)} R_{+}^{(\varrho)}, \rho\right\}\right) \\
+ & \left.D_{0}^{(\alpha \varrho)}\left(2 R_{3}^{(\varrho)} \rho R_{3}^{(\alpha)}-\left\{R_{3}^{(\alpha)} R_{3}^{(\varrho)}, \rho\right\}\right)\right]
\end{aligned}
$$

where $R_{ \pm}^{(1)}=R_{ \pm} \otimes R_{0}$ and $R_{ \pm}^{(2)}=R_{0} \otimes R_{ \pm}$with $R_{-}=|0\rangle\langle 1|$ and $R_{+}=|1\rangle\langle 0|$. Here, $D_{\kappa}^{(\alpha \varrho)}$ and $H_{\kappa}^{(\alpha \varrho)}(\kappa=+,-, 0)$ are determined by the Fourier and Hilbert transforms of the field correlation functions

$$
G_{i j}^{(\alpha \varrho)}(\Delta \tau)=\left\langle\Phi_{i}\left(t_{\alpha}(\tau), \mathbf{x}_{\alpha}(\tau)\right) \Phi_{j}\left(t_{\varrho}\left(\tau^{\prime}\right), \mathbf{x}_{\varrho}\left(\tau^{\prime}\right)\right)\right\rangle
$$

Note here that, $\langle\cdots\rangle$ denotes the expectation value with respect to a certain state of the quantum field. Moreover, since the environment perceived by the quantum system is stationary, the correlation functions of the fields eq. (2.8) are functions of $\Delta \tau=\tau-\tau^{\prime}$. Recalling that the field variables $\phi_{k}^{( \pm)}$in eq. (2.4) are all independent, one finds

$$
G_{i j}^{(\alpha \varrho)}(\Delta \tau)=\sum_{k=1}^{N} \chi_{i}^{k}\left(\chi_{j}^{k}\right)^{*} G^{(\alpha \varrho)}(\Delta \tau)
$$

where $G^{(\alpha \varrho)}(\Delta \tau)$ is the standard Wightman function for a single scalar field. When the scalar field is in the Minkowski vacuum, the explicit form of $G^{(\alpha \varrho)}(\Delta \tau)$ can be written as

$$
G^{(\alpha \varrho)}(\Delta \tau)=-\frac{1}{4 \pi^{2}} \frac{1}{\left[t_{\alpha}(\tau)-t_{\varrho}\left(\tau^{\prime}\right)-i \epsilon\right]^{2}-\left[\mathbf{x}_{\alpha}(\tau)-\mathbf{x}_{\varrho}\left(\tau^{\prime}\right)\right]^{2}} .
$$


We define two functions $\mathcal{G}_{i j}^{(\alpha \varrho)}(x)$ and $\mathcal{K}_{i j}^{(\alpha \varrho)}(x)$ with the Fourier and Hilbert transformations of $G^{(\alpha \varrho)}(\Delta \tau)$ as

$$
\begin{aligned}
\mathcal{G}_{i j}^{(\alpha \varrho)}(x) & =\varepsilon^{2} \int_{-\infty}^{\infty} d t e^{i x t} G_{i j}^{(\alpha \varrho)}(t), \\
\mathcal{K}_{i j}^{(\alpha \varrho)}(x) & =\varepsilon^{2} \int_{-\infty}^{\infty} d t \operatorname{sign}(t) e^{i x t} G_{i j}^{(\alpha \varrho)}(t)=\varepsilon^{2} \frac{P}{\pi i} \int_{-\infty}^{\infty} d \omega \frac{\mathcal{G}_{i j}^{(\alpha \varrho)}(\omega)}{\omega-x},
\end{aligned}
$$

where $P$ denotes principal value. Then, the coefficient matrix $D_{\kappa}^{(\alpha \varrho)}$ can be written explicitly as

$$
D_{ \pm}^{(\alpha \varrho)}=\sum_{i, j=1}^{2}\left(\delta_{i j} \pm i \epsilon_{i j 3}\right) \mathcal{G}_{i j}^{(\alpha \varrho)}( \pm \omega), \quad D_{0}^{(\alpha \varrho)}=\mathcal{G}_{33}^{(\alpha \varrho)}(0) .
$$

Similarly, the coefficients of $H_{\kappa}^{(\alpha \varrho)}$ can be obtained by replacing $\mathcal{G}_{i j}^{(\alpha \varrho)}$ with $\mathcal{K}_{i j}^{(\alpha \varrho)}$ in the above expressions. For the sake of simplicity, we now assume the coefficients $\chi_{\mu}^{a}$ satisfy $\sum_{a=1}^{N} \chi_{\mu}^{a}\left(\chi_{\nu}^{a}\right)^{*}=\delta_{\mu \nu}$, which, for example, can be fulfilled by assuming that the field components $\Phi_{i}(x)$ are independent. Then, $D_{\kappa}^{(\alpha \varrho)}$ can be written explicitly as

$$
D_{ \pm}^{(\alpha \varrho)}=2 \mathcal{G}^{(\alpha \varrho)}( \pm \omega), \quad D_{0}^{(\alpha \varrho)}=\mathcal{G}^{(\alpha \varrho)}(0)
$$

where

$$
\mathcal{G}^{(\alpha \varrho)}(x)=\varepsilon^{2} \int_{-\infty}^{\infty} d t e^{i x t} G^{(\alpha \varrho)}(t)
$$

In the following, we define a set of dimensionless physical parameters using $D_{\kappa}^{(\alpha \varrho)}$ to describe the entanglement dynamics of the quantum system.

(1) $\boldsymbol{\Omega}$. The factor $\Omega$ is defined as

$$
\Omega=\frac{\Gamma^{(\alpha \alpha)}}{\left.\Gamma^{(\alpha \alpha)}\right|_{m=0}}
$$

where $\Gamma^{(\alpha \varrho)}=D_{+}^{(\alpha \varrho)}-D_{-}^{(\alpha \varrho)} . \quad \Omega$ represents the ratio of the spontaneous emission rate of an Unruh-DeWitt detector coupled with massive scalar fields to that of the one coupled with massless scalar fields. Here, the spontaneous emission rate of the Unruh-DeWitt detector coupled to massless scalar fields in the Minkowski vacuum is $\left.\Gamma_{0} \equiv \Gamma^{(11)}\right|_{m=0}=\left.\Gamma^{(22)}\right|_{m=0}=\varepsilon^{2} \omega / \pi$, with $m$ being the mass of the field.

In the acceleration case, we can obtain

$$
\Omega_{a}=\frac{\sinh (\pi \omega / a)}{\pi \omega / a} \frac{m^{2}}{a^{2}}\left[K_{1+i \omega / a}(m / a) K_{-1+i \omega / a}(m / a)-K_{i \omega / a}^{2}(m / a)\right]
$$

where $K_{\nu}(x)$ is the second type of modified Bessel function, and $a$ is the proper acceleration of the detectors. For the convenience of later discussions, $\Omega_{a}$ is written 
as a function of dimensionless variables $m / \omega$ and $a / \omega$, i.e., $\Omega_{a}(m / \omega, a / \omega)$ (See appendix B for the details of the properties of $\Omega_{a}$ ). For comparison, for the thermal bath case, $\Omega_{\beta}$ can be obtained as

$$
\Omega_{\beta}= \begin{cases}\sqrt{1-\frac{m^{2}}{\omega^{2}}}, & \omega>m, \\ 0, & \omega \leq m .\end{cases}
$$

In both the two cases, one can prove that $\Omega \in[0,1]$, with $\Omega=1$ corresponding to the massless case. One can refer to appendix A for the derivations of eqs. (2.17) and (2.18).

(2) $\boldsymbol{\eta}$. If the environment is in an equilibrium state which satisfies the Kubo-MartinSchwinger (KMS) condition [44-46] (which is true for the models considered in the present paper), the Wightman function satisfies the following property

$$
G\left(\Delta \tau-i \beta_{K}\right)=G(-\Delta \tau)
$$

where $\beta_{K}=1 / T_{K}$ is a positive parameter called the inverse KMS temperature. Then, we can obtain that $\mathcal{G}^{+}(-\omega)=e^{-\beta_{K} \omega} \mathcal{G}^{+}(\omega)$, where $\mathcal{G}^{+}(\omega)=\int_{-\infty}^{\infty} G^{+}(\Delta \tau) e^{i \omega \Delta \tau} d \Delta \tau$, and define a dimensionless factor $\eta$ as

$$
\eta=\frac{D_{+}^{(\alpha \varrho)}+D_{-}^{(\alpha \varrho)}}{D_{+}^{(\alpha \varrho)}-D_{-}^{(\alpha \varrho)}}=\frac{e^{\omega \beta_{K}}+1}{e^{\omega \beta_{K}}-1}=\operatorname{coth} \frac{\omega \beta_{K}}{2} .
$$

The factor $\eta$ can be re-expressed with the downward and upward transition rates $\mathcal{R}^{\downarrow}=\mathcal{R}_{|h\rangle \rightarrow|l\rangle}, \mathcal{R}^{\uparrow}=\mathcal{R}_{|l\rangle \rightarrow|h\rangle}$ between any two energy eigenstate $|h\rangle$ (high), and $|l\rangle$ (low) as

$$
\eta=\frac{\mathcal{R}^{\downarrow}+\mathcal{R}^{\uparrow}}{\mathcal{R}^{\downarrow}-\mathcal{R}^{\uparrow}}=2 N_{K}+1=\operatorname{coth} \frac{\omega}{2 T_{K}},
$$

where $N_{K}$ is the effective particle number, and the KMS temperature $T_{K}=1 / \beta_{K}=$ $\omega\left[\ln \left(1+1 / N_{K}\right)\right]^{-1}$. For the acceleration case,

$$
\eta_{a}=\operatorname{coth} \frac{\pi \omega}{a}=\operatorname{coth} \frac{\omega}{2 T_{U}},
$$

where $T_{U}=\frac{a}{2 \pi}$ is the Unruh temperature, and for the thermal bath case

$$
\eta_{\beta}=\operatorname{coth} \frac{\beta \omega}{2}=\operatorname{coth} \frac{\omega}{2 T} .
$$

Here, one can see appendix A for details. Moreover, it is obvious that $\eta \in[1,+\infty]$, with $\eta=1$ corresponding to the case when the (Unruh) temperature is zero.

(3) $\lambda$. In our model, $\Gamma^{(11)}=\Gamma^{(22)}$ and $\Gamma^{(12)}=\Gamma^{(21)}$. Then we can define a factor $\lambda$ as

$$
\lambda=\frac{\left.\Gamma^{(\alpha \varrho)}\right|_{\alpha \neq \varrho}}{\left.\Gamma^{(\alpha \varrho)}\right|_{\alpha=\varrho}}
$$


Similarly, the factor $\lambda$ can be re-expressed with the upward and downward collective transition rates related to the symmetric state $|S\rangle=\frac{1}{\sqrt{2}}(|10\rangle+|01\rangle)$ and antisymmetric state $|A\rangle=\frac{1}{\sqrt{2}}(|10\rangle-|01\rangle)$ as

$$
\lambda=\frac{\mathcal{R}_{S}^{\downarrow}-\mathcal{R}_{A}^{\downarrow}}{\mathcal{R}_{S}^{\downarrow}+\mathcal{R}_{A}^{\downarrow}}=\frac{\mathcal{R}_{S}^{\uparrow}-\mathcal{R}_{A}^{\uparrow}}{\mathcal{R}_{S}^{\uparrow}+\mathcal{R}_{A}^{\uparrow}}
$$

where, the collective rates are respectively $\mathcal{R}_{S}^{\downarrow}=\mathcal{R}_{|E\rangle \rightarrow|S\rangle}=\mathcal{R}_{|S\rangle \rightarrow|G\rangle}, \mathcal{R}_{A}^{\downarrow}=$ $\mathcal{R}_{|E\rangle \rightarrow|A\rangle}=\mathcal{R}_{|A\rangle \rightarrow|G\rangle}, \mathcal{R}_{S}^{\uparrow}=\mathcal{R}_{|G\rangle \rightarrow|S\rangle}=\mathcal{R}_{|S\rangle \rightarrow|E\rangle}$ and $\mathcal{R}_{A}^{\uparrow}=\mathcal{R}_{|G\rangle \rightarrow|A\rangle}=\mathcal{R}_{|A\rangle \rightarrow|E\rangle}$, with $|E\rangle=|11\rangle$ and $|G\rangle=|00\rangle$. From eq. (2.25), it is straightforward to show that $|\lambda| \leq 1$. For the acceleration case, $\lambda_{a}$ can be obtained as

$$
\lambda_{a}=\frac{4 a^{2}}{m^{2}} \frac{\int_{\frac{m}{a}}^{\infty} \frac{1}{a L} \sin \left(a L \sqrt{x^{2}-m^{2} / a^{2}}\right) K_{i 2 \omega / a}(2 x) d x}{K_{1+i \omega / a}(m / a) K_{-1+i \omega / a}(m / a)-K_{i \omega / a}^{2}(m / a)},
$$

which can be written as a function of dimensionless variables, i.e., $\lambda_{a}(m / \omega, a / \omega, L \omega)$. Here, $L$ is the separation between the two detectors. For the thermal bath case,

$$
\lambda_{\beta}=\frac{\sin \left(L \omega \Omega_{\beta}\right)}{L \omega \Omega_{\beta}} .
$$

Recall that $\Omega_{\beta}$ has been given in eq. (2.18). The derivations of eqs. (2.26) and (2.27) are given in appendix $\mathrm{A}$, and the details of the properties of $\lambda_{a}$ are referred to appendix C.

(4) $\zeta^{+}$and $\zeta^{-}$. We define two factors

$$
\zeta^{+}=\frac{D_{0}^{(11)}+D_{0}^{(12)}}{D_{+}^{(11)}+D_{-}^{(11)}}, \quad \zeta^{-}=\frac{D_{0}^{(11)}-D_{0}^{(12)}}{D_{+}^{(11)}+D_{-}^{(11)}}
$$

With the help of eq. (2.14) and the parameters defined previously, $\zeta^{ \pm}$can be reexpressed as

$$
\zeta^{ \pm}=\frac{\mathcal{G}^{(11)}(0) \pm \mathcal{G}^{(12)}(0)}{2\left[\mathcal{G}^{(11)}(\omega)+\mathcal{G}^{(11)}(-\omega)\right]}=\frac{2 T_{K}}{\omega} \frac{1}{\eta} \frac{\Omega(0)}{\Omega(\omega)} \frac{1 \pm \lambda(0)}{2} .
$$

It is obvious that $\zeta^{ \pm} \geq 0$. Now, for the acceleration case, substituting eqs. (2.17), (2.22) and (2.26) into eq. (2.29), one can obtain

$$
\zeta_{a}^{ \pm}=\frac{m^{2}\left[K_{1}^{2}(m / a)-K_{0}^{2}(m / a)\right] \pm 4 a^{2} \int_{\frac{m}{a}}^{\infty} \frac{\sin \left(a L \sqrt{x^{2}-m^{2} / a^{2}}\right)}{a L} K_{0}(2 x) d x}{2 m^{2}\left[K_{1+i \omega / a}(m / a) K_{-1+i \omega / a}(m / a)-K_{i \omega / a}^{2}(m / a)\right] \cosh (\pi \omega / a)},
$$

which can be written as a function of dimensionless variables, i.e., $\zeta_{a}^{ \pm}(m / \omega, a / \omega, L \omega)$ (See appendix D for the details of the properties of $\zeta_{a}^{ \pm}$). Similarly, for the thermal bath case,

$$
\zeta_{\beta}^{+}=\left\{\begin{array}{ll}
\frac{\tanh (\omega \beta / 2)}{\omega \beta / 2}, & m=0, \\
0, & m \neq 0,
\end{array} \quad \zeta_{\beta}^{-}=0 .\right.
$$


With the parameters defined above, $D_{\kappa}^{(\alpha \varrho)}$ in eq. (2.14) can be re-expressed as

$$
\begin{array}{ll}
D_{ \pm}^{(11)}=D_{ \pm}^{(22)}=\frac{1}{2}(\eta \pm 1) \Omega \Gamma_{0}, & D_{ \pm}^{(12)}=D_{ \pm}^{(21)}=\frac{1}{2}(\eta \pm 1) \lambda \Omega \Gamma_{0} \\
D_{0}^{(11)}=D_{0}^{(22)}=\frac{1}{2}\left(\zeta^{+}+\zeta^{-}\right) \eta \Omega \Gamma_{0}, & D_{0}^{(12)}=D_{0}^{(21)}=\frac{1}{2}\left(\zeta^{+}-\zeta^{-}\right) \eta \Omega \Gamma_{0} .
\end{array}
$$

Now, we choose to work in the coupled basis $\{|E\rangle,|S\rangle,|A\rangle,|G\rangle\}$, where $|E\rangle=|11\rangle,|S\rangle=$ $\frac{1}{\sqrt{2}}(|10\rangle+|01\rangle),|A\rangle=\frac{1}{\sqrt{2}}(|10\rangle-|01\rangle)$ and $|G\rangle=|00\rangle$. For simplicity, we assume that the initial density matrix is in the $\mathrm{X}$ form, i.e. the only nonzero elements are those along the diagonal and anti-diagonal of the density matrix in the coupled basis $\{|E\rangle,|S\rangle,|A\rangle,|G\rangle\}$, then the $\mathrm{X}$ form will be maintained during evolution [47]. Thus, a set of equations which describe the time evolution of the density matrix elements can be expressed as

$$
\begin{aligned}
\dot{\mathbf{X}}(\tau) & =-\mathbf{U}\left(\eta, \lambda, \zeta^{-}\right) \mathbf{X}(\tau), & \dot{\rho}_{E G}(\tau)=-\left(1+4 \zeta^{+}\right) \rho_{E G}(\tau), \\
\dot{\rho}_{S A}(\tau) & =-\left(1+2 \zeta^{-}\right) \rho_{S A}(\tau)+2 \zeta^{-} \rho_{A S}(\tau), &
\end{aligned}
$$

where $\rho_{I J}(\tau)=\langle I|\rho(\tau)| J\rangle, I, J \in\{G, E, A, S\}$, and $\dot{\rho}_{I J}(\tau)=d \rho_{I J}(\tau) / d r$ is the derivative with respect to the dimensionless time $r=\eta \Omega \Gamma_{0} \tau$. The column vector $\mathbf{X}(\tau)$ is defined as $\mathbf{X}(\tau)=\left(\begin{array}{llll}\rho_{E}(\tau) & \rho_{S}(\tau) & \rho_{A}(\tau) & \rho_{G}(\tau)\end{array}\right)^{T}$. Here, for brevity, we have abbreviated the diagonal terms of the density matrix elements as $\rho_{I}(\tau)=\rho_{I I}(\tau)$. The coefficient matrix $\mathbf{U}\left(\eta, \lambda, \zeta^{-}\right)$can be expressed as

$$
\mathbf{U}\left(\eta, \lambda, \zeta^{-}\right)=\left(\begin{array}{cccc}
\frac{\eta+1}{\eta} & -\frac{(\eta-1)(1+\lambda)}{2 \eta} & -\frac{(\eta-1)(1-\lambda)}{2 \eta} & 0 \\
-\frac{(\eta+1)(1+\lambda)}{2 \eta} & 1+\lambda+2 \zeta^{-} & -2 \zeta^{-} & -\frac{(\eta-1)(1+\lambda)}{2 \eta} \\
-\frac{(\eta+1)(1-\lambda)}{2 \eta} & -2 \zeta^{-} & 1-\lambda+2 \zeta^{-} & -\frac{(\eta-1)(1-\lambda)}{2 \eta} \\
0 & -\frac{(\eta+1)(1+\lambda)}{2 \eta} & -\frac{(\eta+1)(1-\lambda)}{2 \eta} & \frac{\eta-1}{\eta}
\end{array}\right),
$$

Since $\rho_{G}+\rho_{E}+\rho_{A}+\rho_{S}=1$, only three of the first four equations in eq. (2.33) are independent. The general solution of eq. (2.33) can be written in the following form

$$
\begin{aligned}
\mathbf{X}(\tau) & =\mathbf{M}_{0}(\eta)+\sum_{i=1}^{3} \mathbf{M}_{i}\left(\rho(0), \eta, \lambda, \zeta^{-}\right)[\Theta(\tau)]^{\xi_{i}} \\
\rho_{S A}(\tau) & =\frac{\rho_{S A}(0)+\rho_{S A}(0)}{2} \Theta(\tau)+\frac{\rho_{S A}(0)-\rho_{S A}(0)}{2}[\Theta(\tau)]^{1+4 \zeta^{-}}, \\
\rho_{E G}(\tau) & =\rho_{E G}(0)[\Theta(\tau)]^{1+4 \zeta^{+}}
\end{aligned}
$$

where

$$
\Theta(\tau)=e^{-\eta \Omega \Gamma_{0} \tau},
$$

which ranges from 0 to 1 , and it determines the entanglement evolution with time. Here, $\xi_{i}\left(\eta, \lambda, \zeta^{-}\right)$and $\mathbf{M}_{i}\left(\rho(0), \eta, \lambda, \zeta^{-}\right)$are respectively the eigenvalues and corresponding eigenvectors of the coefficient square matrix $\mathbf{U}\left(\eta, \lambda, \zeta^{-}\right)$. The eigenvalues $\xi_{i}$ satisfy the following equation,

$$
\xi\left[\xi^{3}-4\left(1+\zeta^{-}\right) \xi^{2}+\left(5+12 \zeta^{-}+\lambda^{2} \eta^{-2}-2 \lambda^{2}\right) \xi-2\left(1+4 \zeta^{-}-\lambda^{2}\right)\right]=0 .
$$


Obviously, there is a definite zero root which we label as $\xi_{0}=0$, and the corresponding eigenvector $\mathbf{M}_{0}(\eta)$ can be obtained as

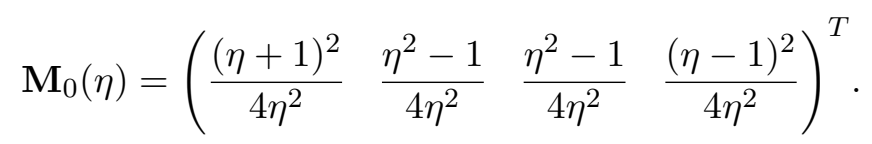

The other three non-negative roots are labeled as $\xi_{1}, \xi_{2}, \xi_{3}$. Hereafter, the subscript " $i$ " of $\xi_{i}$ runs from 1 to 3 except otherwise stated. Note that the explicit expressions for $\xi_{i}\left(\eta, \lambda, \zeta^{-}\right)$and $\mathbf{M}_{i}\left(\rho(0), \eta, \lambda, \zeta^{-}\right)$which are not directly mentioned in the following discussions are not shown here.

We characterize the degree of entanglement by concurrence [48], which ranges from 0 for separable states, to 1 for maximally entangled states. For the X-type states, the concurrence takes the following form [28]

$$
C[\rho(\tau)]=\max \left\{0, K_{1}(\tau), K_{2}(\tau)\right\},
$$

where

$$
\begin{aligned}
& K_{1}(\tau)=\sqrt{\left[\rho_{A}(\tau)-\rho_{S}(\tau)\right]^{2}-\left[\rho_{A S}(\tau)-\rho_{S A}(\tau)\right]^{2}}-2 \sqrt{\rho_{G}(\tau) \rho_{E}(\tau)} \\
& K_{2}(\tau)=2\left|\rho_{G E}(\tau)\right|-\sqrt{\left[\rho_{A}(\tau)+\rho_{S}(\tau)\right]^{2}-\left[\rho_{A S}(\tau)+\rho_{S A}(\tau)\right]^{2}}
\end{aligned}
$$

Substituting eq. (2.35) into eq. (2.39), one can obtain the concurrence $C$, which can be formally written as $C\left[\Omega \tau, \rho(0), \eta, \lambda, \zeta^{ \pm}\right]$.

\section{Entanglement dynamics of a uniformly accelerated quantum system coupled with massive scalar fields}

In this section, we study the entanglement dynamics of a quantum system composed of two uniformly accelerated Unruh-DeWitt detectors coupled with massive scalar fields in the Minkowski vacuum. With the help of the parameters $\left\{\Omega, \eta, \lambda, \zeta^{ \pm}\right\}$defined in the preceding section, we study the time-delay effect (related to the parameter $\Omega$ ), entanglement degradation effect (related to the parameters $\eta, \zeta^{ \pm}$), and entanglement generation effect (related to the parameter $\lambda$ ) in entanglement evolution. We also discuss the Unruh and anti-Unruh effects by comparing the entanglement generated in the acceleration case with that in the thermal bath case.

\subsection{The time-delay effect}

From the time evolution of the density matrix eqs. (2.35) and (2.36), it is obvious that the factor $\Omega$ plays the role of delaying the entanglement evolution, since its range is $0 \leq \Omega \leq 1$. This time-delay effect of the entanglement evolution is general, which is independent of the initial state of the system, or the feature of entanglement evolution (e.g. entanglement generation, degradation, revival, etc). That is, the evolution time for the quantum system coupled with massive fields is always $\Omega^{-1}$ times that of the massless case. This 


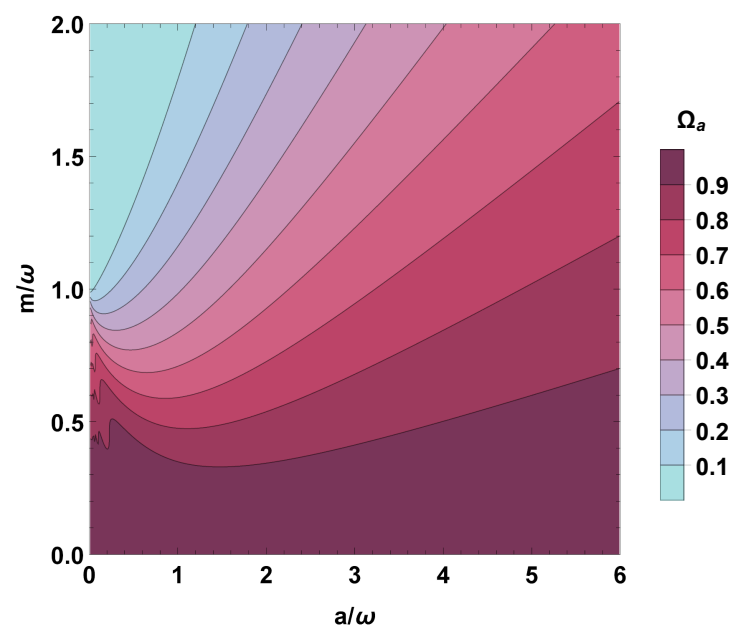

Figure 1. The contour map of the factor $\Omega_{a}$ in parameter space $(a / \omega, m / \omega)$.

time-delay effect is advantageous to entanglement protection, but disadvantageous to entanglement generation.

For uniformly accelerated Unruh-DeWitt detectors coupled to massive scalar fields, the factor $\Omega_{a}$ can be written as a function of $m / \omega$ and $a / \omega$, whose analytical expression and the numerical values are shown in eq. (2.17) and figure 1 respectively. In ref. [38], the properties of $\Omega_{a}(\mathrm{~m} / \omega, a / \omega)$ have been studied in detail. The properties of $\Omega_{a}$ under some limiting conditions, such as the low and high acceleration limit, and the low and high mass limit, are shown in appendix B. In the following, we analyze the influences of the mass of the field and the acceleration on the time-delay effect in entanglement evolution.

\subsubsection{The mass effects}

In this part, we focus on the effects of mass on the time-delay effect. Compared with the massless case, the entanglement evolution in the massive case is generally slower since $\Omega_{a} \leq 1$. When $m \rightarrow 0, \Omega_{a}$ tends to that in the massless case, i.e., $\Omega_{0 a}=1$. Also, it can be proved that $d \Omega_{a} / d m \leq 0$ (See eq. (B.2) in appendix B), so the larger the mass $m$, the slower the evolution. Especially, when $m / \omega \geq 1$, the time delay will increase exponentially. (See appendix B for the details of the properties of $\Omega_{a}$ ). This time-delay phenomenon is shown in figure 2 .

\subsubsection{The acceleration effects}

Comparing $\Omega_{a}$ and $\Omega_{\beta}$ given in eqs. (2.17) and (2.18), one finds that, for uniformly accelerated Unruh-DeWitt detectors in the Minkowski vacuum, $\Omega_{a}$ is not only related to the field mass $m / \omega$, but also to the acceleration $a / \omega$. However, for the static ones in a thermal bath, $\Omega_{\beta}$ is only related to $m / \omega$ but not to the temperature of the bath $T_{U}$ [41]. This leads to significant differences between the two cases.

Firstly, as shown in figure 1 , when $m<\omega$, the factor $\Omega_{a}$ oscillates as the acceleration increases when the acceleration is small, and increases monotonically with the acceleration 

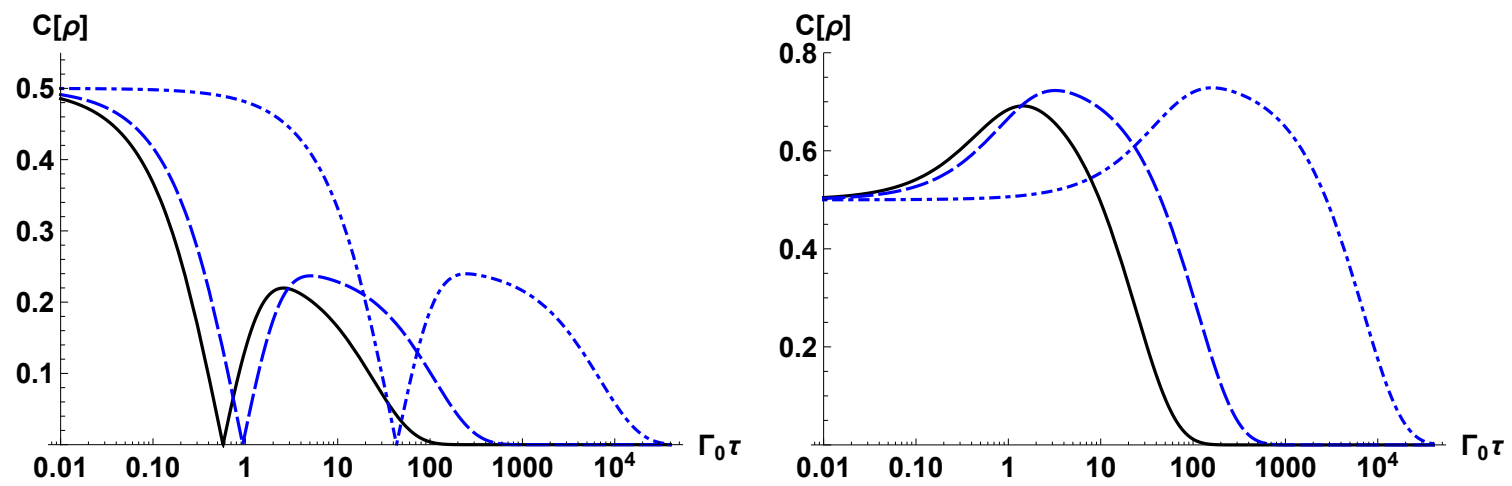

Figure 2. Comparison between the dynamics of concurrence for uniformly accelerated quantum systems coupled with massive scalar fields (blue lines) and massless scalar fields (black lines) initially prepared in $\frac{1}{2}|A\rangle+\frac{\sqrt{3}}{2}|S\rangle$ (left) and $\frac{\sqrt{3}}{2}|A\rangle+\frac{1}{2}|S\rangle$ (right), with $L \omega=0.5, a / \omega=0.1$. The black solid, blue dashed and dot-dashed lines correspond to $m / \omega=0,0.8,1.2$ respectively.
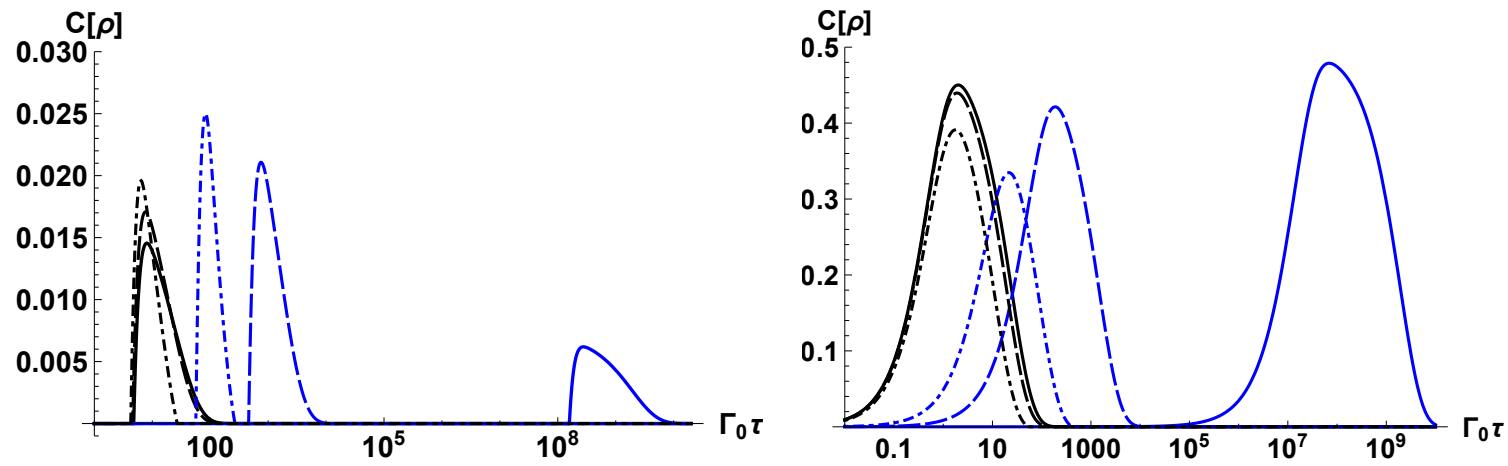

Figure 3. Comparison between the dynamics of concurrence for uniformly accelerated quantum systems coupled with massive scalar fields (blue lines, $m / \omega=2$ ) and massless scalar fields (black lines, $m / \omega=0$ ) initially prepared in $|E\rangle$ (left) and $\frac{\sqrt{2}}{2}|A\rangle \pm \frac{\sqrt{2}}{2}|S\rangle$ (i.e. $|10\rangle$ or $|01\rangle$ ) (right), with $L \omega=0.5$. The solid, dashed and dot-dashed lines correspond to $a / \omega=0.1,0.5,1$ respectively.

when the acceleration is large enough. When $m \geq \omega$, the factor $\Omega_{a}$ increases monotonically with the acceleration. In either case, $\Omega_{a} \rightarrow 1$ when the acceleration tends to infinity (See appendix B for the proof). That is, the time-delay effect brought about by field being massive can be counteracted by a large enough acceleration (See also figure 3). This is in sharp contrast to the case of a static quantum system in a thermal bath, where this time delay brought about by the field being massive is not affected by the temperature. Secondly, in the thermal case, when $m / \omega \geq 1, \Omega_{\beta}=0$, which means that the quantum system is locked up in its initial state $\rho(\tau)=\rho(0)$, and the concurrence is a constant [41]. However, in the acceleration case, as long as $a \neq 0$ and $m \neq \infty$, then $\Omega_{a} \neq 0$, and so the entanglement still evolves, although the evolution may be very slow because $\Omega_{a} \rightarrow 0$ when $a \rightarrow 0$ or $m \rightarrow \infty$. 


\subsection{The entanglement degradation effect}

The entanglement degradation is caused by the dissipation and decoherence due to the quantum system with the environment, which will be discussed in details as follows.

\subsubsection{Entanglement degradation caused by dissipation}

Now, we discuss the entanglement degradation effect caused by dissipation characterized by the factor $\eta$ defined in eq. (2.20), which can be written as $\eta=\operatorname{coth}\left[\omega /\left(2 T_{K}\right)\right]$, where $T_{K}$ is the Unruh temperature in the acceleration case and the environment temperature in the thermal case.

Firstly, the larger the factor $\eta$, the smaller the asymptotic entanglement of the quantum system. When the separation between the two detectors is nonvanishing, $\lambda<1$, and it can be found from eq. (2.37) that the eigenvalues $\xi_{i}$ of the coefficient matrix $\mathbf{U}$ defined in eq. (2.34) are positive. Then, from eq. (2.35), one can obtain the asymptotic state as

$$
\rho_{E}(\infty)=\frac{(\eta-1)^{2}}{4 \eta^{2}}, \quad \rho_{S}(\infty)=\rho_{A}(\infty)=\frac{\eta^{2}-1}{4 \eta^{2}}, \quad \rho_{G}(\infty)=\frac{(\eta+1)^{2}}{4 \eta^{2}},
$$

and $\rho_{I J}(\infty)=0(I \neq J)$. Plugging the asymptotic state into eq. (2.39), we can get

$$
K_{1}(\infty)=K_{2}(\infty)=\frac{1}{2}\left(\eta^{-2}-1\right) \leq 0,
$$

so $C(\infty)=0$, which indicates that the final state is a separable state. When the separation between the two detectors is vanishing, we have $\lambda=1$ and $\zeta^{-}=0$, and the asymptotic state can be obtained as

$$
\begin{array}{ll}
\rho_{E}(\infty)=\frac{(\eta-1)^{2}}{1+3 \eta^{2}}\left[1-\rho_{A}(0)\right], & \rho_{S}(\infty)=\frac{\eta^{2}-1}{1+3 \eta^{2}}\left[1-\rho_{A}(0)\right], \\
\rho_{G}(\infty)=\frac{(\eta+1)^{2}}{1+3 \eta^{2}}\left[1-\rho_{A}(0)\right], & \rho_{A}(\infty)=\rho_{A}(0),
\end{array}
$$

and $\rho_{I J}(\infty)=0(I \neq J)$. Similarly, we can get

$$
\begin{aligned}
& K_{1}(\infty)=\frac{\left|4 \eta^{2} \rho_{A}(0)+1-\eta^{2}\right|-2\left(\eta^{2}-1\right)\left(1-\rho_{A}(0)\right)}{3 \eta^{2}+1} \\
& K_{2}(\infty)=-\frac{2\left(\eta^{2}+1\right) \rho_{A}(0)+\eta^{2}-1}{3 \eta^{2}+1} \leq 0
\end{aligned}
$$

In this case, if $\rho_{A}(0)>\frac{3\left(\eta^{2}-1\right)}{2\left(3 \eta^{2}-1\right)}$, then,

$$
C(\infty)=K_{1}(\infty)=\frac{4\left[1-\rho_{A}(0)\right]}{3 \eta^{2}+1}+2 \rho_{A}(0)-1>0,
$$

which indicates that there exists asymptotic entanglement related to the initial state. It is found that the mass of the field cannot affect the asymptotic entanglement, so the result is the same as that in the massless case [33]. However, this is different from the result when the qubits are accelerating in an environment with nonzero background temperature [49]. 

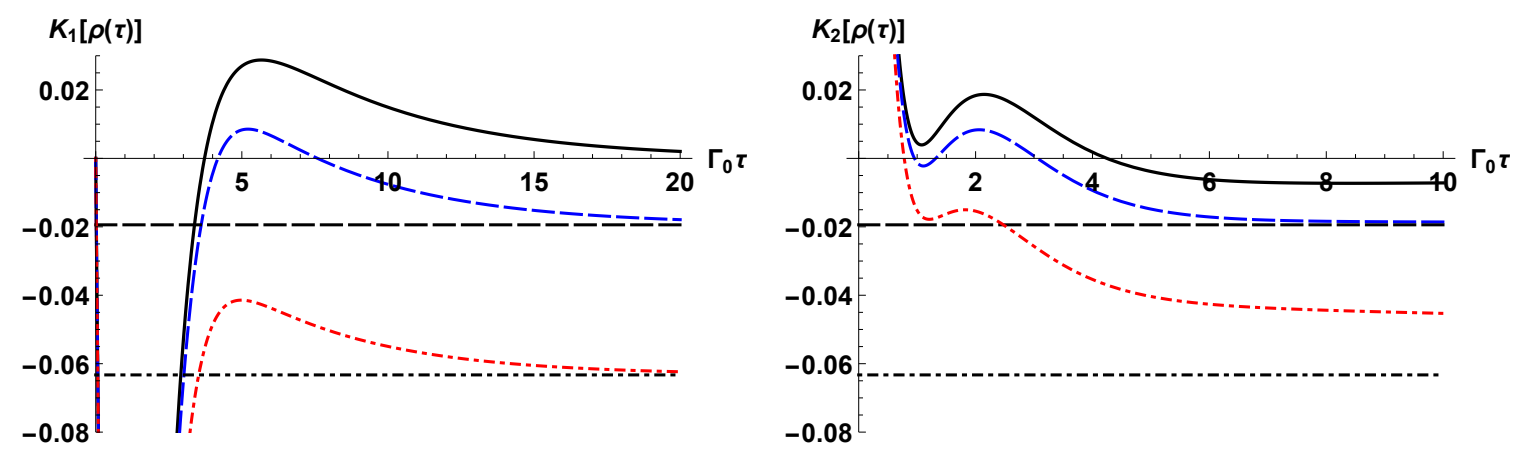

Figure 4. The time evolution of the concurrence parameters $K_{1}$ (left, with $\lambda=0.8, \zeta^{-}=0$ ) and $K_{2}$ (right, with $\lambda=0.98, \zeta^{+}=0$ ) with different dissipation factor $\eta=\operatorname{coth}\left[\omega /\left(2 T_{K}\right)\right]$, for two-atom system prepared in $|E\rangle$ (left), $\sqrt{0.13}|G\rangle+\sqrt{0.87}|E\rangle$ (right). The black solid (horizontal axis), blue (black) dashed and red (black) dot-dashed lines are the evolution-lines (asymptotes) corresponding to $\eta=1,1.02,1.07$ respectively.

From eqs. (3.2) and (3.4), it is easy to prove that $\frac{d K_{1}(\infty)}{d \eta} \leq 0, \frac{d K_{2}(\infty)}{d \eta} \leq 0$, which indicates that $\eta$ plays the role of entanglement degradation.

Secondly, $\eta$ appears in $\Theta(\tau)=e^{-\eta \Omega \Gamma_{0} \tau}$, which shows that it accelerates the evolution of the system. In other words, it speeds up the disentanglement of a quantum system when there is a finite separation between the two detectors.

Thirdly, $\eta$ also affects the value of the eigenvector $\mathbf{M}_{i}\left(\rho(0), \eta, \lambda, \zeta^{-}\right)$(i.e., the amplitude functions in eq. (2.35)) and the eigenvalues $\xi_{i}\left(\eta, \lambda, \zeta^{-}\right)$of the coefficient matrix $\mathbf{U}\left(\eta, \lambda, \zeta^{-}\right)$, which affect the phenomena of entanglement evolution, such as entanglement creation, revival, enhancement, etc. We show the effect of $\eta$ on the concurrence coefficients $K_{1}$ and $K_{2}$ defined in eq. (2.40) in figure 4 . There one can see that the larger the $\eta$ (the temperature $T_{K}$ ), the more the curve of $K_{1(2)}$ moves downwards, and the smaller the concurrence is. So the phenomena of entanglement generation, enhancement, and revival will not show up when the factor $\eta$ becomes large enough.

To summarize, the factor $\eta$, which describes the thermal dissipative effect of the environment, plays the role of degrading entanglement in entanglement dynamics. It is only related to the Unruh temperature $T_{U}=\frac{a}{2 \pi}$ in the acceleration case and the environment temperature $T$ in the thermal case. This indicates that, from the aspect of entanglement degradation, an accelerated quantum system in the Minkowski vacuum suffers the same dissipative effect as that of a static one in a thermal bath at the Unruh temperature, as expected due to the Unruh effect.

\subsubsection{Entanglement degradation caused by decoherence}

From the evolution equations of quantum systems (2.33), it can be found that the factors $\zeta^{ \pm}$defined in eq. (2.28) play the role of decoherence. We show that the decoherence factors cause an additional entanglement degradation in the following. 
With the help of the evolution equations of density matrix elements (2.33), the first derivative of $K_{1(2)}(\tau)$ versus time $\tau$ can be written as

$$
\begin{aligned}
& \frac{d K_{1}(\tau)}{d \tau}=\left[-\eta K_{1}(\tau)-4 \zeta^{-} \eta K_{11}(\tau)-f_{1}(\tau, \eta)+\lambda\left[\rho_{A}(\tau)-\rho_{S}(\tau)\right] h_{1}(\tau, \eta)\right] \Omega \Gamma_{0}, \\
& \frac{d K_{2}(\tau)}{d \tau}=\left[-\eta K_{2}(\tau)-4 \zeta^{+} \eta K_{21}(\tau)-f_{2}(\tau, \eta)+\lambda\left[\rho_{S}(\tau)-\rho_{A}(\tau)\right] h_{2}(\tau, \eta)\right] \Omega \Gamma_{0},
\end{aligned}
$$

where the functions $f_{i}(\tau, \eta)$ and $h_{i}(\tau, \eta)$ can be expressed as

$$
\begin{aligned}
& f_{1}(\tau, \eta)=\frac{\left[(\eta+1) \rho_{E}(\tau)+(\eta-1) \rho_{G}(\tau)\right]\left[\rho_{A}(\tau)+\rho_{S}(\tau)\right]}{K_{12}(\tau)}, \\
& f_{2}(\tau, \eta)=\frac{\left[(\eta+1) \rho_{E}(\tau)+(\eta-1) \rho_{G}(\tau)\right]\left[\rho_{A}(\tau)+\rho_{S}(\tau)\right]}{K_{22}(\tau)} .
\end{aligned}
$$

and

$$
\begin{aligned}
& h_{1}(\tau, \eta)=\frac{\eta\left[\rho_{A}(\tau)+\rho_{S}(\tau)\right]}{K_{11}(\tau)}+\frac{K_{1}(\tau)\left[(\eta+1) \rho_{E}(\tau)+(\eta-1) \rho_{G}(\tau)\right]}{K_{11}(\tau) K_{12}(\tau)} \\
& h_{2}(\tau, \eta)=\frac{\eta\left[\rho_{A}(\tau)+\rho_{S}(\tau)\right]}{K_{22}(\tau)}
\end{aligned}
$$

with

$$
\begin{aligned}
& K_{11}(\tau)=\sqrt{\left[\rho_{A}(\tau)-\rho_{S}(\tau)\right]^{2}-\left[\rho_{A S}(\tau)-\rho_{S A}(\tau)\right]^{2}}, \\
& K_{12}(\tau)=2 \sqrt{\rho_{E}(\tau) \rho_{G}(\tau)}, \\
& K_{21}(\tau)=2 \sqrt{\rho_{E G}(\tau) \rho_{G E}(\tau)}, \\
& K_{22}(\tau)=\sqrt{\left[\rho_{A}(\tau)+\rho_{S}(\tau)\right]^{2}-\left[\rho_{A S}(\tau)+\rho_{S A}(\tau)\right]^{2}} .
\end{aligned}
$$

It can be found from eq. (3.6) that, the decoherence factors $\zeta^{-}$and $\zeta^{+}$contribute an additional negative rate of change of $K_{1}(\tau)$ and $K_{2}(\tau)$ respectively, which means that the decoherence factors $\zeta^{ \pm}$bring an additional entanglement degradation. Moreover, from eq. (3.6), one can see that the larger the factors $\zeta^{ \pm}$, the stronger the entanglement degradation. Unlike the dissipation factor $\eta$, which is only related to the acceleration for accelerated detectors coupled with massive scalar field, the decoherence factors $\zeta_{a}^{ \pm}(m / \omega, a / \omega, L \omega)$ are related to the acceleration $a$, the mass of the field $m$, and the separation between the detectors $L$. In the following, we focus on the effects of acceleration and mass on the entanglement degradation brought about by the decoherence factors $\zeta^{ \pm}$in details.

The acceleration effects The decoherence factors $\zeta_{\beta}^{ \pm}$are always zero for a two-detector system coupled with massive scalar field in a thermal bath (see eq. (2.31)), while they are nonzero in the acceleration case (see eq. (2.30)). This indicates that the accelerated detectors are subjected to an additional disentangling effect brought about by the decoherence factors $\zeta^{ \pm}$compared with those immersed in a thermal bath.

When the acceleration approaches zero, both the decoherence factors $\zeta_{a}^{ \pm}$and $\zeta_{\beta}^{ \pm}$are vanishing. (See appendix D for the details of the properties of $\zeta_{a}^{ \pm}$). In figure 5, we plot the 

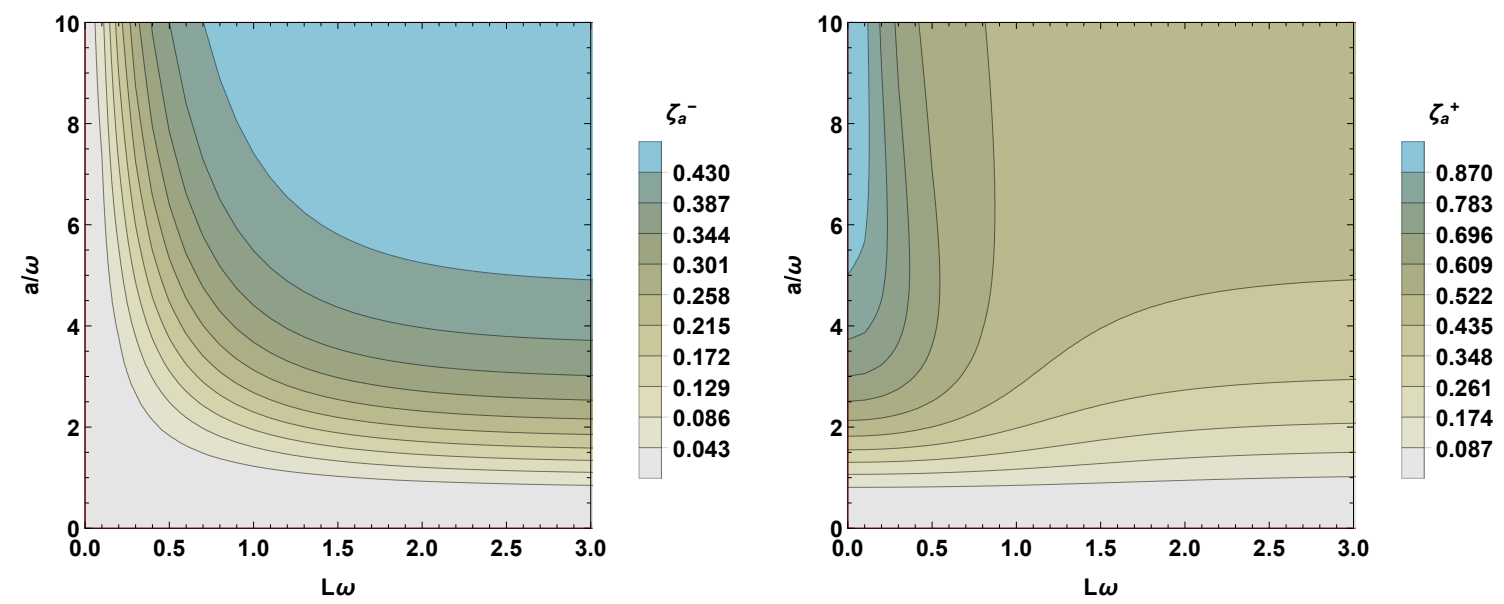

Figure 5. The contour maps of the decoherence factors $\zeta_{a}^{-}$(left) and $\zeta_{a}^{+}$(right) in parameter space $(L \omega, a / \omega)$ for two accelerated Unruh-DeWitt detectors coupled with massive scalar fields $(m / \omega=1)$.

contour maps of the decoherence factors $\zeta_{a}^{-}$(left) and $\zeta_{a}^{+}$(right) in the parameter space $(L \omega, a / \omega)$ for two accelerated Unruh-DeWitt detectors coupled with massive scalar fields. It can be found that, the factor $\zeta_{a}^{-}$increases monotonically with acceleration, while the relation between the factor $\zeta_{a}^{+}$and acceleration is non-monotonic when the separation of the two detectors is relatively small compared with the transition wavelength.

The mass effects For detectors coupled with massless scalar fields, the decoherence factors in the acceleration case $\zeta_{a}^{ \pm}$and the thermal bath case $\zeta_{\beta}^{ \pm}$can be respectively written as

$$
\zeta_{a}^{ \pm}=\frac{\tanh (\pi \omega / a)}{\pi \omega / a} \frac{1}{2}\left[1 \pm \frac{2 \sinh ^{-1}(a L / 2)}{a L \sqrt{1+a^{2} L^{2} / 4}}\right], \quad \zeta_{\beta}^{+}=\frac{\tanh (\beta \omega / 2)}{\beta \omega / 2}, \quad \zeta_{\beta}^{-}=0 .
$$

When the fields the detectors coupled to are changed from massless ones to massive ones, the decoherance factor $\zeta^{ \pm}$disappears for the thermal bath case, but not for the acceleration case. In particular, we obtain that (See appendix D for details)

$$
\begin{aligned}
& \lim _{m \rightarrow \infty} \zeta_{a}^{+}(m / \omega, a / \omega, L \omega)= \begin{cases}\operatorname{sech}(\pi \omega / a), & L=0, \\
\frac{1}{2} \operatorname{sech}(\pi \omega / a), & L \neq 0,\end{cases} \\
& \lim _{m \rightarrow \infty} \zeta_{a}^{-}(m / \omega, a / \omega, L \omega)= \begin{cases}0, & L=0, \\
\frac{1}{2} \operatorname{sech}(\pi \omega / a), & L \neq 0 .\end{cases}
\end{aligned}
$$

That is, even if the mass of the field goes to infinity, the decoherance factors $\zeta_{a}^{ \pm}$are nonzero as long as the separation between the detectors is non-vanishing. To show more details, in figure 6 , we plot the contour maps of the decoherence factors $\zeta_{a}^{-}$(left) and $\zeta_{a}^{+}$(right) in the parameter space $(L \omega, m / \omega)$ for two accelerated Unruh-DeWitt detectors coupled with massive scalar fields. As shown in figure 6 , the factor $\zeta_{a}^{+}$decreases monotonically with the mass of the field, while the relation between the factor $\zeta_{a}^{-}$and the mass of the field is monotonic only when the separation between the detectors is large enough. This 

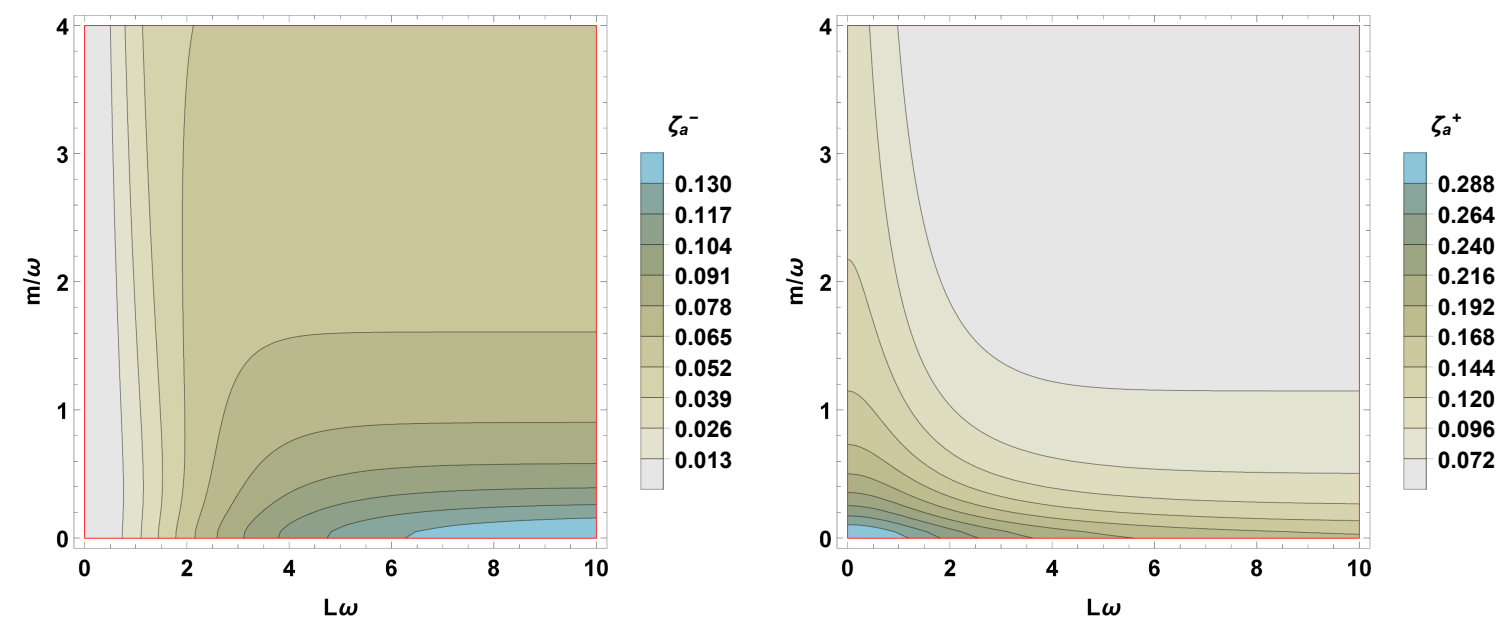

Figure 6. The contour maps of the decoherence factors $\zeta_{a}^{-}$(left) and $\zeta_{a}^{+}$(right) in parameter space of $(L \omega, m / \omega)$ for two accelerated $(a / \omega=1)$ Unruh-DeWitt detectors coupled with massive scalar fields.

indicates that the mass of the field must weaken the additional entanglement degradation effect brought about by the factor $\zeta_{a}^{+}$; however, it can both strengthen and weaken the effect brought about by the factor $\zeta_{a}^{-}$.

In conclusion, accelerated detectors not only suffer the same dissipation effect as that caused by a thermal bath, but also an additional decohernece effect, both of which contribute to entanglement degradation.

\subsection{The entanglement generation effect}

The entanglement generation effect is crucially dependent on the factor $\lambda(|\lambda| \leq 1)$ defined in eq. (2.24). When $\lambda=0$, it can be obtained from eq. (3.6) that $d C(\tau) / d \tau \leq 0$. That is, when $\lambda=0$, it is impossible to create entanglement when the system is initially separable, and the entanglement can only decrease during evolution when the system is initially entangled. In fact, from eq. (3.6), one can find that a large enough $|\lambda|$ is a necessary condition for entanglement generation.

In the following, we investigate the entanglement generation of a quantum system with the initial state $|E\rangle$. We focus on the relationship between entanglement creation and the factor $\lambda$. In figure 7 , we show the maximum of concurrence $C_{\max }$ during evolution in the parameter space $(\lambda, \eta)$ with different decoherence factor $\zeta^{-}$. Here only the $\lambda>0$ part is shown, because it is symmetric about $\lambda=0$. It is shown that, for each dissipative factor $\eta$ and decoherence factor $\zeta^{-}$, there exists a range of $\lambda \in\left(\lambda_{\min }, \lambda_{\max }\right)$ within which entanglement can be created. For the acceleration case, this range is related to the field mass $m$, the acceleration $a$ and the separation $L$, while for the thermal case, it is related to the temperature of the bath $T$ only. One can find from figure 7 that, the lower limit $\lambda_{\text {min }}$ increases as $\eta$ and $\zeta^{-}$increase.

For a quantum system composed of two uniformly accelerated Unruh-DeWitt detectors coupled with massive scalar field in the Minkowski vacuum, the coherence factor $\lambda_{a}$ can be 

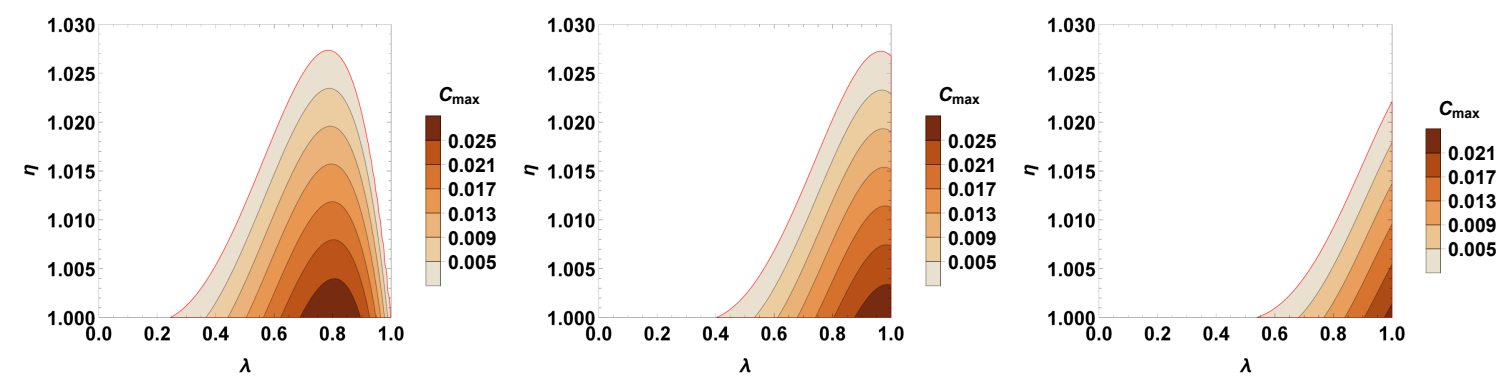

Figure 7. The contour maps of the maximum of concurrence during evolution $C_{\max }$ in parameter space $(\lambda, \eta)$ with $\zeta^{-}=0$ (left), 0.1 (middle), and 0.2 (right) for quantum systems initially prepared in $|E\rangle$. Note that contour lines with $C_{\max }<0.001$ are not drawn here.
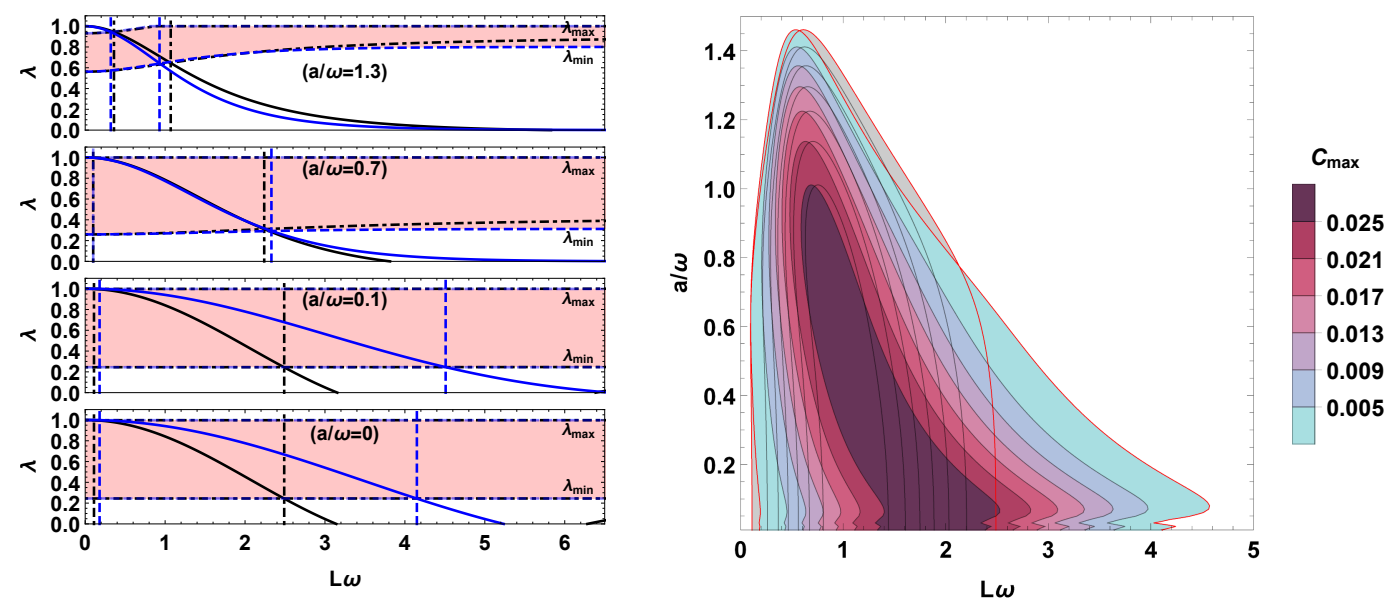

Figure 8. (Left) The factor $\lambda$ as a function of separation $L \omega$, with different accelerations $a / \omega$. The horizontal blue-dashed and black-dot-dashed lines mark the upper and lower limits $\lambda_{\max }$ and $\lambda_{\min }$ within which entanglement can be generated $\left(C_{\max } \geq 0.001\right)$. (Right) Contour map of the maximal concurrence during evolution for quantum systems prepared in $|E\rangle$ in the parameter space $(L \omega, a / \omega)$. The figures show the differences between the results in the massive case $(m / \omega=0.8$, the blue lines on the left, and the colored contour on the right) and the massless case (the black lines on the left, and the un-colored contour on the right).

written as $\lambda_{a}(m / \omega, a / \omega, L \omega)$, whose explicit expression has been given in eq. (2.26), and the expressions in several limiting cases have been shown in appendix C. Now, we focus on how the mass of the field and the acceleration affect the parameter regions within which the system can get entangled.

\subsubsection{The mass effects}

In this part, we study the parameter regions within which entanglement creation can occur for two uniformly accelerated Unruh-DeWitt detectors prepared in $|E\rangle$ coupled with massive scalar fields, and compare the result with that in the massless case. We examine two situations, namely $m \geq \omega$ (figure 8 ) and $m<\omega$ (figure 9).

Firstly, in figures 8 and 9 , it is shown that, compared with the massless case, the region of separation $L \omega$ within which entanglement can be created is expanded when the 

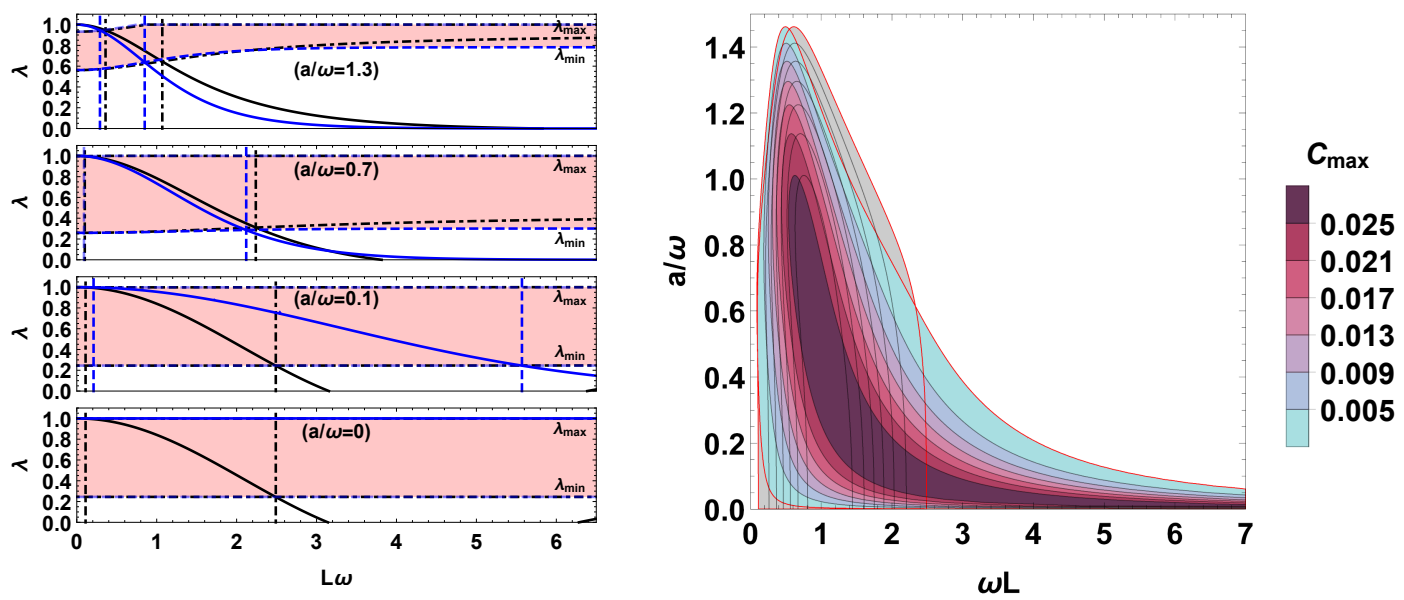

Figure 9. (Left) The factor $\lambda$ as a function of separation $L \omega$, with different accelerations $a / \omega$. The horizontal blue-dashed and black-dot-dashed lines mark the upper and lower limits $\lambda_{\max }$ and $\lambda_{\min }$ within which entanglement can be generated $\left(C_{\max } \geq 0.001\right)$. (Right) Contour map of the maximal concurrence during evolution for quantum systems prepared in $|E\rangle$ in the parameter space $(L \omega, a / \omega)$. The figures show the differences between the results in the massive case $(\mathrm{m} / \omega=1.2$, the blue lines on the left, and the colored contour on the right) and the massless case (the black lines on the left, and the un-colored contour on the right).

acceleration $a / \omega$ is smaller than a critical value $a_{\text {crit }} / \omega$ determined by the field mass $m / \omega$, and is compressed when $a>a_{\text {crit }}$. This is distinct from the fact that the region of separation for entanglement generation is always expanded for a static quantum system coupled with massive fields (in vacuum or in a thermal bath), compared with the massless case [41].

Secondly, by comparing figure 8 and figure 9 , we find that the behaviors of the factor $\lambda_{a}$ at $a \rightarrow 0$ is completely different for $m<\omega$ and $m>\omega$, resulting in a significant difference in the possible regions of separation $L \omega$ for entanglement creation. In the acceleration case, when the mass of the field exceeds the energy level spacing of the Unruh-DeWitt detectors, long-distance entanglement creation can be achieved when the acceleration is small, in contrast to the static case in which the mass of the field has to be smaller than but close to the energy level spacing in order to achieve long-distance entanglement [41].

\subsubsection{The acceleration effects}

Now we focus on the effects of acceleration on entanglement evolution, and compare the results with those of static ones in a thermal bath at the Unruh temperature. First, according to the analytical expressions eqs. (2.26) to (2.27), in the acceleration case, the factor $\lambda_{a}$ is a function of the acceleration $a$, while in the thermal case, $\lambda_{\beta}$ is independent of temperature, which results in essential differences between the two cases.

In figure 10, it is shown that when $a / \omega=0$, the acceleration case is the same as the static case as expected. When the acceleration increases, in a certain region where the acceleration is relatively small, the possible region of separation $L \omega$ for entanglement generation varies oscillatorily from being compressed to being enlarged compared with that in the thermal case, due to the oscillation of $\lambda_{a}$ with acceleration $a$. The amplitude of this 

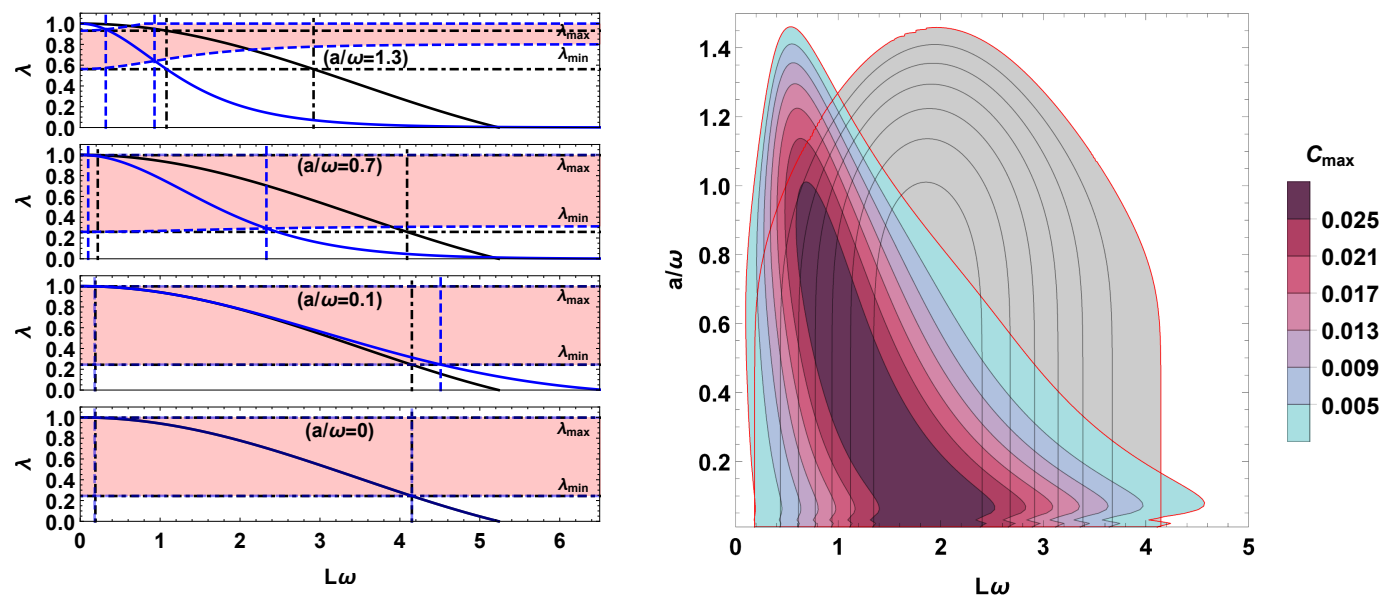

Figure 10. (Left) The factor $\lambda$ as a function of separation $L \omega$, with different accelerations $a / \omega$. The horizontal blue-dashed and black-dot-dashed lines mark the upper and lower limits $\lambda_{\max }$ and $\lambda_{\min }$ within which entanglement can be generated $\left(C_{\max } \geq 0.001\right)$. (Right) Contour map of the maximal concurrence during evolution for quantum systems prepared in $|E\rangle$ with $m / \omega=0.8$ in the parameter space $(L \omega, a / \omega)$. The figures show the differences between the results in the acceleration case (the blue lines on the left, and the colored contour on the right) and the thermal case (the black lines on the left, and the un-colored contour on the right).

oscillation increases with acceleration, until the maximal amplitude is reached (at about $a / \omega \approx 0.1$ in figure 10 ), which causes a maximal expansion of the possible region of separation $L \omega$ for entanglement generation. Then, $\lambda_{a}$ decays monotonically with acceleration, which causes the compression of the possible region of separation $L \omega$ for entanglement generation compared with that of the thermal case, as shown in figure 10. However, in the thermal case, since $\lambda_{\beta}$ is independent of temperature, the possible region of separation $L \omega$ for entanglement generation decreases monotonically as temperature increases.

In figure 11, we study the effects of mass on entanglement dynamics for two uniformly accelerated Unruh-DeWitt detectors, and compare the results with those of in the thermal case. From figure 11, we draw the following conclusions: ${ }^{1}$

1. When $m / \omega<1$, in the thermal case, the damping of $\lambda_{\beta}$ with $L \omega$ gradually slows down as $m / \omega$ increases. When $m / \omega \rightarrow 1, \lambda_{\beta}$ becomes a constant $\left(\lambda_{\beta}=1\right)$. As a result, the region of $L \omega$ within which entanglement can be generated is significantly enlarged when $m / \omega$ is close to 1 . However, in the acceleration case, $\lambda_{a}$ does not approach a constant no matter how large the mass is. Therefore, the regions of $L \omega$ within which the two detectors can be entangled are only slightly enlarged.

2. When $m / \omega \geq 1$, there are significant differences between the acceleration case and the thermal case. For the thermal case, the factor $\Omega_{\beta}$ equals to 0 , so the detectors are locked up in the initial state as if it were a closed system, and entanglement generation cannot occur. However, in the acceleration case, no matter how large

\footnotetext{
${ }^{1}$ In figure 11 , the acceleration is set to $a / \omega=0.1$. However, the conclusions still hold if a larger acceleration is chosen.
} 

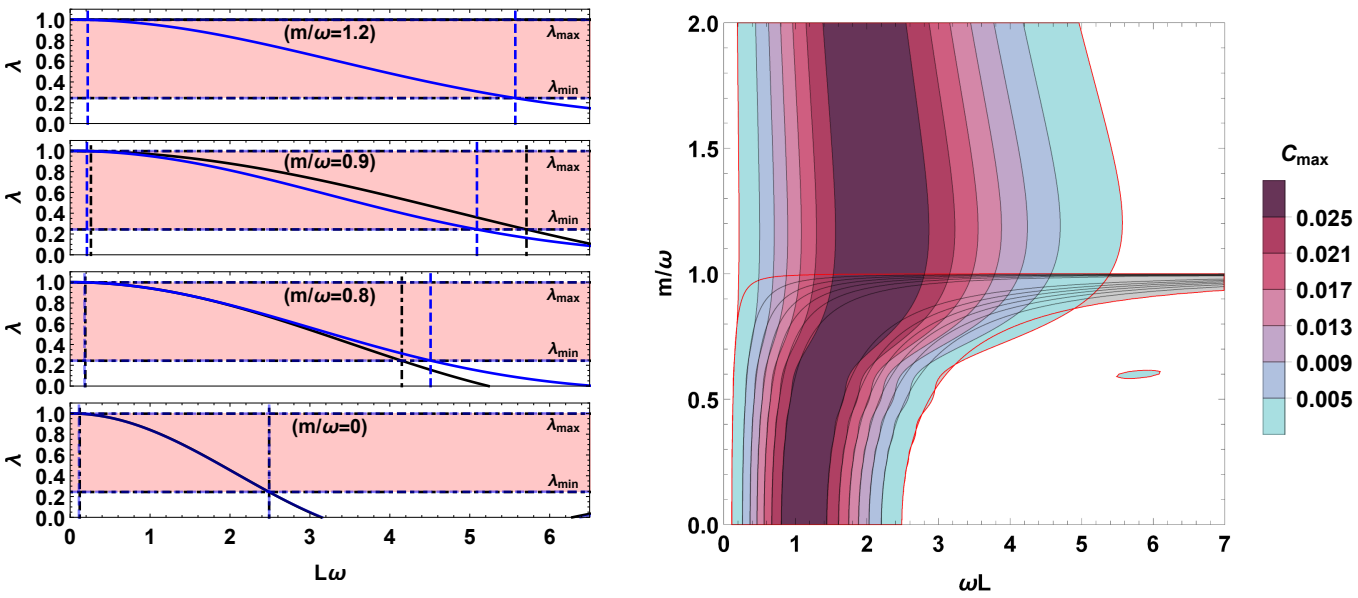

Figure 11. (Left) The factor $\lambda$ as a function of separation $L \omega$, with different mass $m / \omega$. The horizontal blue-dashed and black-dot-dashed lines mark the upper and lower limits $\lambda_{\max }$ and $\lambda_{\min }$ within which entanglement can be generated $\left(C_{\max } \geq 0.001\right)$. (Right) Contour map of the maximal concurrence during evolution for quantum systems prepared in $|E\rangle$ with $a / \omega=0.1$ in the parameter space $(L \omega, m / \omega)$. The figures show the differences between the results in the acceleration case (the blue lines on the left, and the colored contour on the right) and the thermal case (the black lines on the left, and the un-colored contour on the right).

$m / \omega$ is (as long as it is not infinite, see eq. (C.4)), $\lambda_{a}$ is a function of $L \omega$ ranging from 0 to 1 , and the factor $\Omega_{a} \neq 0$. Therefore, entanglement generation is possible for certain $L \omega$ (but it may take a long time since the factor $\Omega_{a}$ decays exponentially as $m / \omega$ increases). A similar conclusion has been drawn in ref. [50], in which it has been found that a single detector relaxes to its thermal equilibrium state extremely inefficiently in the large mass limit.

\subsection{The Unruh and anti-Unruh effects}

The Unruh effect can be sensed by a quantum system coupled with the vacuum fields, i.e. an Unruh-DeWitt detector $[3,5]$. For static detectors in a thermal bath, it is well-known that the higher the temperature, the more often the detector clicks. Since the Unruh temperature is proportional to the proper acceleration [3], it is expected the larger the acceleration, the more often the Unruh-DeWitt detector clicks. However, in certain cases, the transition rate of a uniformly accelerated detector may decrease with acceleration in some parameter regimes, e.g. in the presence of a boundary [8], when the field the detector coupled with is massive [38], and when the duration of the detector-field coupling is finite [39] (but long enough to satisfy the KMS condition [44-46]). This phenomenon is named as the anti-Unruh effect in ref. [39], and is further divided into two categories [40], i.e. the strong anti-Unruh effect (the effective excitation-to-deexcitation ratio (EDR) temperature of a detector decreases as the KMS temperature increases), and the weak anti-Unruh effect (a detector clicks less often as the KMS temperature increases), which is a necessary condition for the strong one. Recently, it is found that the anti-Unruh effect introduced in refs. $[39,40]$ may possibly be viewed as an amplification mechanism for quantum en- 

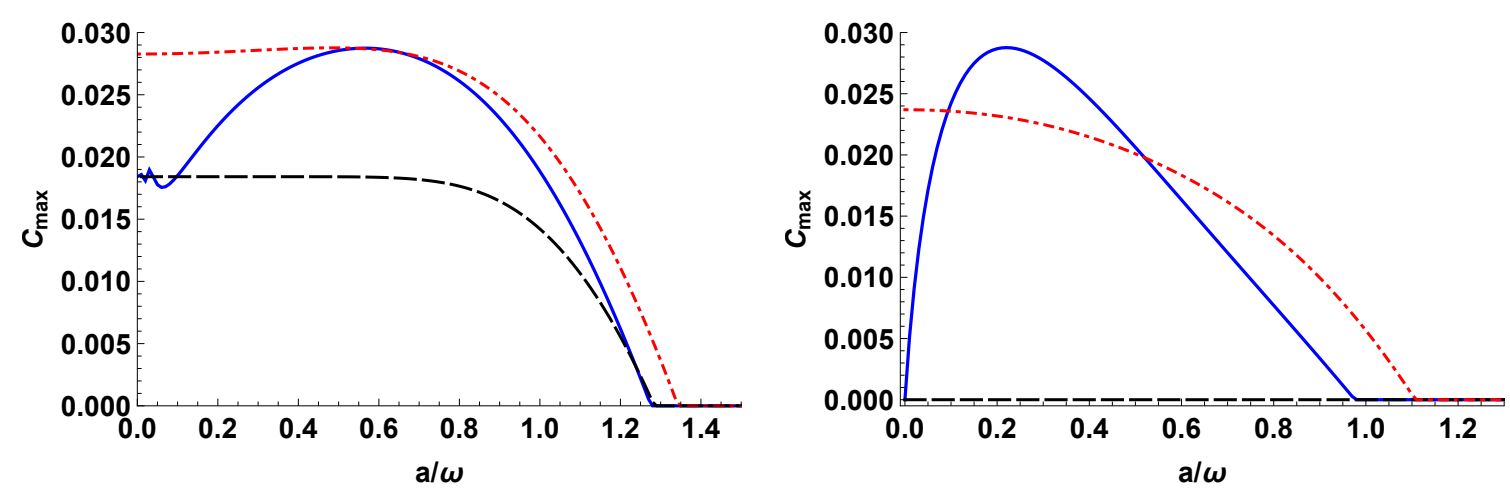

Figure 12. Comparison between the maximum of concurrence during evolution for uniformly accelerated quantum systems coupled with massive (blue solid lines) and massless (red dot-dashed lines), and static ones coupled with massive scalar fields in a thermal bath at the Unruh temperature (black dashed lines) initially prepared in $|E\rangle$. Here, $m / \omega=0.8, L \omega=1.0$ (left), and $m / \omega=1.2$, $L \omega=1.5$ (right).

tanglement within a certain parameter regime [51]. Moreover, an anti-Unruh phenomenon in the transition probabilities of two entangled uniformly accelerated atoms in a thermal bath has been shown in ref. [52].

In ref. [34], we have shown that, for a pair of two-level detectors coupled with massless scalar fields, the maximal concurrence during evolution may increase with acceleration for specific inter-detector separations, in contrast to the fact that it always decreases monotonically with temperature in the thermal case. This may also be called as an anti-Unruh phenomenon in terms of the entanglement generated. In the massive case, as shown in the contour maps (figures 8 and 9), for any inter-detector separation, the maximal concurrence quantifying the entanglement generated during evolution may increase with acceleration when the acceleration is relatively small with respect to the energy level spacing of the detectors. Therefore, in contrast to the massless case in which the anti-Unruh phenomenon appears only for specific separation, it is a general phenomenon in the massive case.

As an example, in figure 12, we plot the maximal concurrence as a function of acceleration $a / \omega$ with fixed mass $m / \omega$ and separation $L \omega$. As shown in figure 12 (left), when $m / \omega<1$, for small accelerations $(a / \omega<0.57)$, the maximal concurrence oscillates with acceleration since the entanglement creation effect from $\lambda$ which oscillates with acceleration is stronger than the entanglement degradation effect from $\eta$. However, when the acceleration is large enough $(a / \omega>0.57)$, the maximal concurrence decreases monotonically with acceleration. When $m / \omega>1$, since $\lambda$ decays monotonically with acceleration from 1 (when $a / \omega=0$ ) to 0 (when $a / \omega \rightarrow \infty$ ), the maximal concurrence varies from 0 (when $a / \omega=0$ ) to a certain non-zero maximum value (when $a / \omega=0.22$ ), and then to 0 (when $a / \omega>0.98$ ), as shown in figure 12 (right). In either case, the anti-Unruh phenomenon occurs when the acceleration is relatively small with respect to the energy level spacing of the detectors $(a / \omega<0.57$ in the first case, and $a / \omega<0.22$ in the second case).

Based on the previous discussions, the Unruh and anti-Unruh effect in terms of entanglement dynamics can be understood as follows. 
Firstly, from the perspective of dissipation (related to $\eta$ ), an accelerated quantum system in the Minkowski vacuum suffers the same dissipative effect as that of a static one in a thermal bath at the Unruh temperature. As a result, the quantum system composed of two accelerated Unruh-DeWitt detectors will be driven into an asymptotic thermal state without entanglement when the separation between the two detectors is nonvanishing. This is what one expects based on the Unruh effect [1-4].

Secondly, from the perspective of entanglement generation, the factor $\lambda$ which affects entanglement generation is independent of temperature for static detectors in a thermal bath, while it is related to acceleration for accelerated detectors.

This shows that acceleration contributes to both the entanglement degradation and creation, while the temperature of a thermal bath contributes to the entanglement degradation only. Therefore, if the entanglement creation effect overweighs the entanglement degradation effect, the maximum entanglement that can be generated during evolution may not decrease monotonically with the Unruh temperature as it does in the thermal bath case. This explains why the anti-Unruh phenomenon in terms of the entanglement appears.

\section{Summary}

In this paper, we have investigated, in the framework of open quantum systems, the entanglement dynamics of a quantum system composed of two uniformly accelerated UnruhDeWitt detectors coupled with fluctuating massive scalar fields in the Minkowski vacuum. We first define a set of parameters, which play the roles of time delay, dissipation, decoherence and entanglement generation in the evolution process. With the help of these parameters, we study the influences of the mass of the field and the acceleration on the entanglement dynamics through comparing the corresponding results in the massless case and the thermal bath case respectively. The relevant conclusions are as follows.

Firstly, on the entanglement evolution rate, the entanglement evolution process for a quantum system coupled with massive fields is always slower compared with that of the one coupled with massless fields, which is advantageous to entanglement protection, but disadvantageous to entanglement generation. Also, this time-delay effect brought about by the field being massive can however be counteracted by a large enough acceleration. It is interesting to note, however, that in the thermal case, this time delay is not affected by the temperature. In particular, when the mass of the field is greater than the energy level spacing of the detectors, static detectors in a thermal bath will be locked up in its initial state, while entanglement generation is still possible in the acceleration case.

Secondly, on the entanglement degradation, we find that there are two effects causing entanglement degradation, namely the dissipation effect and decoherence effect. The accelerated detectors not only suffer the same dissipation effect as that caused by a thermal bath, but also an additional decoherence effect, both of which contribute to entanglement degradation.

Thirdly, on the entanglement creation, the region of spatial separation between the detectors within which entanglement can be generated is always enlarged for a static quantum system coupled with massive fields in a thermal bath compared with that in the massless case, while it can be both compressed and enlarged in the acceleration case. 
Fourthly, on the asymptotic entanglement, two accelerated Unruh-DeWitt detectors will be driven into an asymptotic thermal state without entanglement when the separation between the two detectors is nonvanishing, or an asymptotic entangled state related to its initial state when the separation is vanishing, regardless of whether the mass of the field is greater or less than the energy level spacing of the detectors. However, for the thermal bath case, the detectors will be locked up in the initial state when the mass of the field is larger than the energy level spacing of the detectors, thus preserving the initial entanglement if it exists.

Finally, in conclusion, the entanglement dynamics for uniformly accelerated detectors coupled with massive fields is essentially different from that of the static ones in a thermal bath at the Unruh temperature. In particular, the maximal concurrence of the quantum system generated during evolution may increase with acceleration when the acceleration is relatively small compared with the energy level spacing of the detectors for any interdetector separation, in contrast to the monotonic decreases of the maximal concurrence with temperature for static detectors in a thermal bath, which can be considered as an anti-Unruh effect in terms of the entanglement generated.

\section{Acknowledgments}

This work was supported in part by the NSFC under Grants No.11805063, No. 11690034, and No. 12075084, and the Hunan Provincial Natural Science Foundation of China under Grant No. 2020JJ3026.

\section{A The expressions of the entanglement dynamic parameters in various cases}

For the sake of simplicity, we assume that the coefficients $\chi_{\mu}^{k}$ defined in eq. (2.4) satisfy $\sum_{k=1}^{N} \chi_{\mu}^{k}\left(\chi_{\nu}^{k}\right)^{*}=\delta_{\mu \nu}$. Then, $G_{i j}^{(\alpha \varrho)}(\Delta \tau)=\delta_{i j} G^{(\alpha \varrho)}(\Delta \tau)$, where $G^{(\alpha \varrho)}(\Delta \tau)$ is the standard Wightman function which can be expressed as

$$
G^{(\alpha \varrho)}(\Delta \tau)=\left\langle\phi\left(t_{\alpha}(\tau), \mathbf{x}_{\alpha}(\tau)\right) \phi\left(t_{\varrho}\left(\tau^{\prime}\right), \mathbf{x}_{\varrho}\left(\tau^{\prime}\right)\right)\right\rangle .
$$

In the free Minkowski spacetime, the scalar field operator $\phi(t, \mathbf{x})$ can be expanded as

$$
\phi(t, \mathbf{x})=\int d^{3} k \frac{1}{\sqrt{2 \omega_{k}\left(2 \pi^{3}\right)}}\left[a_{k} e^{i \mathbf{k x}-i \omega_{k} t}+a_{k}^{\dagger} e^{-i \mathbf{k x}+i \omega_{k} t}\right],
$$

where $a_{k}$ and $a_{k}^{\dagger}$ are the annihilation and creation operators for field quanta with frequency $\omega_{k}$ and momentum $k=|\mathbf{k}|$ satisfying the dispersion relation $\omega_{k}^{2}=k^{2}+m^{2}$, with $m$ being mass of the scalar fields. Substituting eq. (A.2) into eq. (A.1), we obtain

$$
G^{(\alpha \varrho)}(\Delta \tau)=\frac{1}{4 \pi^{2}} \int_{m}^{\infty} \frac{\sin \left(\sqrt{\omega_{k}^{2}-m^{2}}\left|\Delta \vec{x}_{\alpha \varrho}\right|\right)}{\left|\Delta \vec{x}_{\alpha \varrho}\right|}\left[2\left\langle N_{\omega_{k}}\right\rangle \cos \left(\omega_{k} \Delta t_{\alpha \varrho}\right)+e^{-i \omega_{k} \Delta t_{\alpha \varrho}}\right] d \omega_{k},
$$

where $\left|\Delta \vec{x}_{\alpha \varrho}\right|=\sqrt{\left(x_{\alpha}-x_{\varrho}^{\prime}\right)^{2}+\left(y_{\alpha}-y_{\varrho}^{\prime}\right)^{2}+\left(z_{\alpha}-z_{\varrho}^{\prime}\right)^{2}}, \Delta t_{\alpha \varrho}=t_{\alpha}-t_{\varrho}^{\prime}$, and $N_{\omega_{k}}=a_{k}^{\dagger} a_{k}$ is the particle number operator. 


\section{A.1 The acceleration case}

For a uniformly accelerated quantum system composed of two Unruh-DeWitt detectors with a separation $L$ perpendicular to the acceleration, the trajectories of each detector are

$$
\begin{aligned}
& t_{1}(\tau)=\frac{1}{a} \sinh a \tau, \quad x_{1}(\tau)=\frac{1}{a} \cosh a \tau, \quad y_{1}(\tau)=0, \quad z_{1}(\tau)=0, \\
& t_{2}(\tau)=\frac{1}{a} \sinh a \tau, \quad x_{2}(\tau)=\frac{1}{a} \cosh a \tau, \quad y_{2}(\tau)=0, \quad z_{2}(\tau)=L,
\end{aligned}
$$

where $a$ is the proper acceleration of the detectors. Substituting the trajectories above into eqs. (A.3) and (2.14), and using the following integral formula

$$
\int_{-\infty}^{+\infty} e^{-i\left(2 \omega_{k} / a\right) \sinh (a u / 2)} e^{i \omega u} d u=(4 / a) e^{\pi \omega / a} K_{i 2 \omega / a}\left(2 \omega_{k} / a\right),
$$

one obtains

$$
\begin{aligned}
& D_{ \pm}^{(\alpha \varrho)}=\frac{\varepsilon^{2} \omega}{\pi} \frac{2 e^{ \pm \pi \omega / a}}{\pi \omega / a} \int_{\frac{m}{a}}^{\infty} \frac{\sin \left(a\left|\Delta z_{\alpha \varrho}\right| \sqrt{x^{2}-m^{2} / a^{2}}\right)}{a\left|\Delta z_{\alpha \varrho}\right|} K_{i 2 \omega / a}(2 x) d x, \\
& D_{0}^{(\alpha \varrho)}=\frac{\varepsilon^{2} a}{\pi^{2}} \int_{\frac{m}{a}}^{\infty} \frac{\sin \left(a\left|\Delta z_{\alpha \varrho}\right| \sqrt{x^{2}-m^{2} / a^{2}}\right)}{a\left|\Delta z_{\alpha \varrho}\right|} K_{0}(2 x) d x .
\end{aligned}
$$

Here, $\left|\Delta z_{\alpha \varrho}\right|=L$ when $\alpha \neq \varrho$, and $\left|\Delta z_{\alpha \varrho}\right|=0$ when $\alpha=\varrho$. According to the definitions of $\Omega, \eta$ and $\lambda$, which have been shown in eqs. (2.16), (2.20) and (2.24) respectively, we can obtain that

$$
\begin{aligned}
\Omega_{a} & =\frac{\sinh (\pi \omega / a)}{\pi \omega / a} \frac{m^{2}}{a^{2}}\left[K_{1+i \omega / a}(m / a) K_{-1+i \omega / a}(m / a)-K_{i \omega / a}^{2}(m / a)\right], \\
\eta_{a} & =\operatorname{coth} \frac{\pi \omega}{a} \\
\lambda_{a} & =\frac{4 a^{2}}{m^{2}} \frac{\int_{\frac{m}{a}}^{\infty} \frac{1}{a L} \sin \left(a L \sqrt{x^{2}-m^{2} / a^{2}}\right) K_{i 2 \omega / a}(2 x) d x}{K_{1+i \omega / a}(m / a) K_{-1+i \omega / a}(m / a)-K_{i \omega / a}^{2}(m / a)} .
\end{aligned}
$$

Here, the subscript " $a$ " in $\Omega_{a}, \eta_{a}$ and $\lambda_{a}$ denotes the acceleration case.

\section{A.2 The thermal bath case}

For two static Unruh-DeWitt detectors in a thermal bath at temperature $T=\frac{1}{\beta},\left\langle N_{\omega_{k}}\right\rangle=$ $1 /\left(e^{\omega_{k} / T}-1\right)=1 /\left(e^{\beta \omega_{k}}-1\right)$. Plugging eq. (A.3) into eq. (2.14), we obtain

$$
D_{ \pm}^{(\alpha \varrho)}= \begin{cases}\frac{\varepsilon^{2} \omega}{2 \pi} \frac{\sin \left(\sqrt{\omega^{2}-m^{2}}\left|\Delta z_{\alpha \varrho}\right|\right)}{\omega\left|\Delta z_{\alpha \varrho}\right|}\left(\operatorname{coth} \frac{\omega}{2 T} \pm 1\right), & \omega>m \\ 0, & 0 \leq \omega \leq m\end{cases}
$$

and

$$
D_{0}^{(\alpha \varrho)}= \begin{cases}\frac{\varepsilon^{2} T}{2 \pi}, & m=0 \\ 0, & m \neq 0 .\end{cases}
$$

Then, direct calculations show that

$$
\Omega_{\beta}=\left\{\begin{array}{ll}
\sqrt{1-\frac{m^{2}}{\omega^{2}}}, & \omega>m, \\
0, & \omega \leq m .
\end{array} \quad \eta_{\beta}=\operatorname{coth} \frac{\beta \omega}{2}=\operatorname{coth} \frac{\omega}{2 T}, \quad \lambda_{\beta}=\frac{\sin \left(L \omega \Omega_{\beta}\right)}{L \omega \Omega_{\beta}} .\right.
$$

Here, the subscript " $\beta$ " in $\Omega_{\beta}, \eta_{\beta}$ and $\lambda_{\beta}$ denotes the thermal bath case. 


\section{B The limit properties of $\Omega_{a}(m / \omega, a / \omega)$}

The factor $\Omega_{a}$ in eq. (2.17) can be obtained as follows,

$$
\begin{aligned}
\Omega_{a} & =\frac{4 \sinh (\pi \omega / a)}{\pi \omega / a} \int_{0}^{\infty} \frac{k^{2}}{\sqrt{k^{2}+m^{2} / a^{2}}} K_{i 2 \omega / a}\left(2 \sqrt{k^{2}+m^{2} / a^{2}}\right) d k \\
& =\frac{2 \sinh (\pi \omega / a)}{\pi \omega / a} \int_{0}^{\infty} k K_{i \omega / a}^{2}\left(\sqrt{k^{2}+m^{2} / a^{2}}\right) d k \\
& =\frac{\sinh (\pi \omega / a)}{\pi \omega / a} \frac{m^{2}}{a^{2}}\left[K_{1+i \omega / a}(m / a) K_{-1+i \omega / a}(m / a)-K_{i \omega / a}^{2}(m / a)\right],
\end{aligned}
$$

which can be written as a function of dimensionless variables as $\Omega_{a}(m / \omega, a / \omega)$. Furthermore, it can be obtained that

$$
\frac{d \Omega_{a}}{d m}=-\frac{2 \sinh (\pi \omega / a)}{\pi \omega / a} \frac{m}{a^{2}} K_{i \omega / a}^{2}(m / a) \leq 0 .
$$

In the following, we discuss the limit properties of $\Omega_{a}(m / \omega, a / \omega)$.

\section{B.1 The low-mass limit}

When $m \ll \omega$ and $m \ll a, \Omega_{a}(m / \omega, a / \omega)$ can be approximated by,

$$
\Omega_{a}(m / \omega, a / \omega) \approx 1-\frac{m^{2}}{2 \omega^{2}}\left[1+\left(1+\frac{\omega^{2}}{a^{2}}\right)^{-\frac{1}{2}} \cos \left(2 \frac{\omega}{a} \ln \frac{m}{2 a}-\varphi(\omega / a)\right)\right],
$$

where $\varphi(\alpha)$ is an argument function defined as

$$
\varphi(\alpha)=\arg \left[(1+i \alpha) \Gamma^{2}(i \alpha)\right]
$$

Note that $\Gamma(z)$ is the Euler gamma function. This shows that the mass-dependent term gives a small correction which is negative. When the mass of the scalar field approaches 0 ,

$$
\lim _{m \rightarrow 0} \Omega_{a}(m / \omega, a / \omega)=1
$$

and the result reduces to that in the massless case as expected.

\section{B.2 The high-mass limit}

When the mass of scalar field is much larger than the energy level spacing and the acceleration, i.e. $m \gg \omega$ and $m \gg a, \Omega_{a}(m / \omega, a / \omega)$ can be approximated by,

$$
\Omega_{a}(m / \omega, a / \omega) \approx e^{-2 \frac{m}{a}} \frac{\sinh (\pi \omega / a)}{2 \omega / a} .
$$

For a fixed acceleration, it exponentially approaches to zero as the mass of the field increases. Thus,

$$
\lim _{m \rightarrow \infty} \Omega_{a}(m / \omega, a / \omega)=0 .
$$




\section{B.3 The low-acceleration limit}

In the low-acceleration limit, i.e. the acceleration is much smaller than the energy level spacing and the field mass, $a \ll \omega$ and $a \ll m, \Omega_{a}(m / \omega, a / \omega)$ can be approximately written as (for a detailed derivation see ref. [38])

$$
\Omega_{a}(m / \omega, a / \omega) \approx \begin{cases}\sqrt{1-(m / \omega)^{2}}\left[1+\cos \left(2 A_{1} \omega / a\right)\left(\frac{1}{72}+B\right) \frac{a}{A_{1} \omega}\right], & \omega>m, \\ \frac{1}{2} \sqrt{(m / \omega)^{2}-1}\left(\frac{25}{72}+B\right) \frac{a}{A_{2} \omega} e^{-2 A_{2} \omega / a}, & \omega \leq m .\end{cases}
$$

Here, $A_{1}, A_{2}$ and $B$ are positive, which are defined respectively as

$$
\begin{aligned}
A_{1} & =\ln \frac{\omega+\sqrt{\omega^{2}-m^{2}}}{m}-\sqrt{1-(m / \omega)^{2}}, \\
A_{2} & =\sqrt{(m / \omega)^{2}-1}-\arccos (\omega / m), \\
B & =\left(1+C^{2} / 3\right) A C / 4,
\end{aligned}
$$

where $C=1 / \sqrt{1-m^{2} / \omega^{2}}$ and $A=A_{1}$, for $\omega>m$; and $C=1 / \sqrt{m^{2} / \omega^{2}-1}$ and $A=A_{2}$, for $\omega \leq m$. So, when the acceleration $a$ approaches zero,

$$
\lim _{a \rightarrow 0} \Omega_{a}(m / \omega, a / \omega)= \begin{cases}\sqrt{1-(m / \omega)^{2}}, & \omega>m, \\ 0, & \omega \leq m,\end{cases}
$$

which is the results of a static quantum system in vacuum [41], as expected.

\section{B.4 The high-acceleration limit}

For the high-acceleration limit where $a \gg \omega$ and $a \gg m$, keeping only the lowest-order correction term, one finds that

$$
\Omega_{a}(m / \omega, a / \omega) \approx 1-\frac{m^{2}}{a^{2}}\left[\left(\ln \frac{m}{a}\right)^{2}-1.23 \ln \frac{m}{a}+0.63\right],
$$

which gives

$$
\lim _{a \rightarrow \infty} \Omega_{a}(m / \omega, a / \omega)=1
$$

\section{The limit properties of $\lambda_{a}(m / \omega, a / \omega, L \omega)$}

For the acceleration case, the factor $\lambda$ defined in eq. (2.24) can be expressed in the following form

$$
\begin{aligned}
\lambda_{a} & =\frac{\int_{0}^{\infty} \frac{k}{\sqrt{k^{2}+m^{2} / a^{2}}} K_{i 2 \omega / a}\left(2 \sqrt{k^{2}+m^{2} / a^{2}}\right) \frac{\sin (a L k)}{a L} d k}{\int_{0}^{\infty} \frac{k^{2}}{\sqrt{k^{2}+m^{2} / a^{2}}} K_{i 2 \omega / a}\left(2 \sqrt{k^{2}+m^{2} / a^{2}}\right) d k} \\
& =\frac{\int_{0}^{\infty} k K_{i \omega / a}^{2}\left(\sqrt{k^{2}+m^{2} / a^{2}}\right) J_{0}(a L k) d k}{\int_{0}^{\infty} k K_{i \omega / a}^{2}\left(\sqrt{k^{2}+m^{2} / a^{2}}\right) d k} \\
& =\frac{4 a^{2}}{m^{2}} \frac{\int_{\frac{m}{a}}^{\infty} \frac{1}{a L} \sin \left(a L \sqrt{x^{2}-m^{2} / a^{2}}\right) K_{i 2 \omega / a}(2 x) d x}{K_{1+i \omega / a}(m / a) K_{-1+i \omega / a}(m / a)-K_{i \omega / a}^{2}(m / a)}
\end{aligned}
$$


where $J_{\nu}(x)$ and $K_{\nu}(x)$ are the Bessel function of the first type and the modified Bessel function of the second type, respectively. The factor $\lambda_{a}$ can be written as a function of dimensionless variables as $\lambda_{a}(m / \omega, a / \omega, L \omega)$.

\section{C.1 The low-mass limit}

Keeping only the lowest-order term, in the low-mass limit where $m \ll \omega$ and $m \ll a$, the limit of $\lambda_{a}$ when $m \rightarrow 0$ is

$$
\lim _{m \rightarrow 0} \lambda_{a}(m / \omega, a / \omega, L \omega)=\frac{\sin \left(\frac{2 \omega}{a} \sinh ^{-1} \frac{a L}{2}\right)}{L \omega \sqrt{1+a^{2} L^{2} / 4}},
$$

which reduces to the result in the massless case.

\section{C.2 The high-mass limit}

When $m \gg a$ and $m \gg \omega, \lambda_{a}$ can be approximated as

$$
\lambda_{a}(m / \omega, a / \omega, L \omega) \approx \frac{e^{-\left(\sqrt{4+a^{2} L^{2}}-2\right) \frac{m}{a}}}{1+a^{2} L^{2} / 4} .
$$

Therefore, in the limit of $m \rightarrow \infty, \lambda_{a}$ is dependent on the value of $a L$.

$$
\lim _{m \rightarrow \infty} \lambda_{a}(m / \omega, a / \omega, L \omega)= \begin{cases}1, & a L=0 \\ 0, & a L \neq 0 .\end{cases}
$$

\section{C.3 The low-acceleration limit}

If $a \ll m, a \ll \omega$ and $a \ll 1 / L$,

$$
\lim _{a \rightarrow 0} \lambda_{a}(m / \omega, a / \omega, L \omega)= \begin{cases}\frac{\sin \left(L \sqrt{\omega^{2}-m^{2}}\right)}{L \sqrt{\omega^{2}-m^{2}}}, & m / \omega<1, \\ 1, & m / \omega \geq 1,\end{cases}
$$

which reduces to the result in the vacuum case eq. (2.27).

\section{C.4 The high-acceleration limit}

When $a \gg m, \lambda_{a}$ can be approximated as

$$
\lambda_{a}(m / \omega, a / \omega, L \omega) \approx \frac{\sin \left(\frac{2 \omega}{a} \sinh ^{-1} \frac{a L}{2}\right)}{L \omega \sqrt{1+a^{2} L^{2} / 4}},
$$

which is the result in the massless case. Based on this, if $a \gg \omega$, it can further be approximate as,

$$
\lambda_{a}(m / \omega, a / \omega, L \omega) \approx \frac{4 \sinh ^{-1}\left(\frac{a L}{2}\right)}{a L \sqrt{a^{2} L^{2}+4}} .
$$

Furthermore, if $a \gg 1 / L$, then

$$
\lambda_{a}(m / \omega, a / \omega, L \omega) \approx \frac{4 \log (a L)}{a^{2} L^{2}} .
$$


Here, if $a L \ll 1$, the approximate expression of $\lambda_{a}$ can be written as $\lambda_{a} \approx 1-a^{2} L^{2} / 6$. Thus, we can get the limit of $\lambda_{a}$ when $a \rightarrow \infty$ as

$$
\lim _{a \rightarrow \infty} \lambda_{a}(m / \omega, a / \omega, L \omega)= \begin{cases}1, & L=0, \\ 0, & L \neq 0 .\end{cases}
$$

\section{The limit properties of $\zeta_{a}^{ \pm}(m / \omega, a / \omega, L \omega)$}

For the acceleration case, the factors $\zeta^{ \pm}$defined in eq. (2.28) can be expressed in the following form

$$
\zeta_{a}^{ \pm}=\frac{m^{2}\left[K_{1}^{2}(m / a)-K_{0}^{2}(m / a)\right] \pm 4 a^{2} \int_{\frac{m}{a}}^{\infty} \frac{\sin \left(a L \sqrt{x^{2}-m^{2} / a^{2}}\right)}{a L} K_{0}(2 x) d x}{2 m^{2}\left[K_{1+i \omega / a}(m / a) K_{-1+i \omega / a}(m / a)-K_{i \omega / a}^{2}(m / a)\right] \cosh (\pi \omega / a)},
$$

where $K_{\nu}(x)$ is the modified Bessel function of the second type. The factor $\zeta_{a}^{ \pm}$can be written as a function of dimensionless variables as $\zeta_{a}^{ \pm}(m / \omega, a / \omega, L \omega)$.

\section{D.1 The low-mass limit}

Keeping only the lowest-order term, in the low-mass limit where $m \ll \omega$ and $m \ll a$, the limit of $\zeta_{a}^{ \pm}$when $m \rightarrow 0$ is

$$
\lim _{m \rightarrow 0} \zeta_{a}^{ \pm}(m / \omega, a / \omega, L \omega)=\frac{\tanh (\pi \omega / a)}{\pi \omega / a} \frac{1}{2}\left[1 \pm \frac{2 \sinh ^{-1}(a L / 2)}{a L \sqrt{1+a^{2} L^{2} / 4}}\right],
$$

which reduces to the result in the massless case.

\section{D.2 The high-mass limit}

When $m \gg a$ and $m \gg \omega$, with the help of eqs. (B.6) and (C.3), $\zeta_{a}^{ \pm}$can be approximated as

$$
\zeta_{a}^{ \pm}(m / \omega, a / \omega, L \omega) \approx \frac{1}{2 \cosh (\pi \omega / a)}\left[1 \pm \frac{e^{-\left(\sqrt{4+a^{2} L^{2}}-2\right) \frac{m}{a}}}{1+a^{2} L^{2} / 4}\right] .
$$

Therefore, in the limit of $m \rightarrow \infty, \zeta_{a}^{ \pm}$is dependent on the value of $L$.

$$
\begin{aligned}
& \lim _{m \rightarrow \infty} \zeta_{a}^{+}(m / \omega, a / \omega, L \omega)= \begin{cases}\operatorname{sech}(\pi \omega / a), & L=0, \\
\frac{1}{2} \operatorname{sech}(\pi \omega / a), & L \neq 0,\end{cases} \\
& \lim _{m \rightarrow \infty} \zeta_{a}^{-}(m / \omega, a / \omega, L \omega)= \begin{cases}0, & L=0, \\
\frac{1}{2} \operatorname{sech}(\pi \omega / a), & L \neq 0 .\end{cases}
\end{aligned}
$$

\section{D.3 The low-acceleration limit}

If $a \ll m, a \ll \omega$ and $a \ll 1 / L$, with the help of eqs. (B.8) and (C.5), one can find that

$$
\zeta_{a}^{ \pm}(m / \omega, a / \omega, L \omega) \approx \begin{cases}\frac{1}{2} \frac{\tanh (\pi \omega / a)}{\pi \omega / a}(1 \pm 1), & m / \omega=0, \\ \frac{\pi}{4} \frac{\tanh (\pi \omega / a)}{\pi \omega / a} \frac{e^{-2 m / a}}{\sqrt{1-m^{2} / \omega^{2}}}(1 \pm 1), & 0<m / \omega<1, \\ \frac{\pi}{8} \frac{\tanh (\pi \omega / a)}{\pi \omega / a} \frac{A_{2} \omega}{a} \frac{e^{-2\left(m-\omega A_{2}\right) / a}}{\sqrt{m^{2} / \omega^{2}-1}\left(\frac{25}{72}+B\right)}(1 \pm 1), & m / \omega \geq 1,\end{cases}
$$


Here, $A_{2}$ and $B$ have been defined in eq. (B.9). Then, from the approximate expression (D.6), one can obtain that

$$
\lim _{a \rightarrow 0} \zeta_{a}^{ \pm}(m / \omega, a / \omega, L \omega)=0 .
$$

\section{D.4 The high-acceleration limit}

When $a \gg m, a \gg \omega$, taking eqs. (B.12) and (C.9) into eq. (2.29), we can get the limit of $\zeta_{a}^{ \pm}$when $a \rightarrow \infty$ as

$$
\begin{aligned}
& \lim _{a \rightarrow \infty} \zeta_{a}^{+}(m / \omega, a / \omega, L \omega)= \begin{cases}1, & L=0, \\
\frac{1}{2}, & L \neq 0 .\end{cases} \\
& \lim _{a \rightarrow \infty} \zeta_{a}^{-}(m / \omega, a / \omega, L \omega)= \begin{cases}0, & L=0, \\
\frac{1}{2}, & L \neq 0 .\end{cases}
\end{aligned}
$$

Open Access. This article is distributed under the terms of the Creative Commons Attribution License (CC-BY 4.0), which permits any use, distribution and reproduction in any medium, provided the original author(s) and source are credited.

\section{References}

[1] S.A. Fulling, Nonuniqueness of canonical field quantization in Riemannian space-time, Phys. Rev. D 7 (1973) 2850 [inSPIRE].

[2] P.C.W. Davies, Scalar particle production in Schwarzschild and Rindler metrics, J. Phys. A 8 (1975) 609 [INSPIRE].

[3] W.G. Unruh, Notes on black hole evaporation, Phys. Rev. D 14 (1976) 870 [InSPIRE].

[4] L.C.B. Crispino, A. Higuchi and G.E.A. Matsas, The Unruh effect and its applications, Rev. Mod. Phys. 80 (2008) 787 [arXiv:0710.5373] [INSPIRE].

[5] B. DeWitt, in General Relativity: An Einstein Centenary Survey, edited by S.W. Hawking and W. Israel, Cambridge University Press, Cambridge U.K. (1980).

[6] S. Takagi, Vacuum Noise and Stress Induced by Uniform Acceleration: Hawking-Unruh Effect in Rindler Manifold of Arbitrary Dimension, Prog. Theor. Phys. Suppl. 88 (1986) 1 [INSPIRE].

[7] J. Audretsch and R. Müller, Spontaneous excitation of an accelerated atom: The Contributions of vacuum fluctuations and radiation reaction, Phys. Rev. A 50 (1994) 1755 [gr-qc/9408019] [INSPIRE].

[8] H.W. Yu and S. Lu, Spontaneous excitation of an accelerated atom in a spacetime with a reflecting plane boundary, Phys. Rev. D 72 (2005) 064022 [Erratum ibid. 73 (2006) 109901] [gr-qc/0509053] [INSPIRE].

[9] Z. Zhu, H.W. Yu and S. Lu, Spontaneous excitation of an accelerated hydrogen atom coupled with electromagnetic vacuum fluctuations, Phys. Rev. D 73 (2006) 107501 [gr-qc/0604116] [INSPIRE].

[10] J. Audretsch and R. Müller, Radiative energy shifts of accelerated atoms, Phys. Rev. A 52 (1995) 629 [gr-qc/9503058] [INSPIRE]. 
[11] R. Passante, Radiative level shifts of an accelerated hydrogen atom and the Unruh effect in quantum electrodynamic, Phys. Rev. A 57 (1998) 1590.

[12] L. Rizzuto et al., Nonthermal effects of acceleration in the resonance interaction between two uniformly accelerated atoms, Phys. Rev. A 94 (2016) 012121 [arXiv:1601.04502] [INSPIRE].

[13] W. Zhou, R. Passante and L. Rizzuto, Resonance interaction energy between two accelerated identical atoms in a coaccelerated frame and the Unruh effect, Phys. Rev. D 94 (2016) 105025 [arXiv: 1609.06931] [INSPIRE].

[14] L. Rizzuto, Casimir-Polder interaction between an accelerated two-level system and an infinite plate, Phys. Rev. A 76 (2007) 062114.

[15] Z. Zhu and H.W. Yu, Position dependent energy level shifts of an accelerated atom in the presence of a boundary, Phys. Rev. A 82 (2010) 042108 [arXiv:1009.1425] [InSPIRE].

[16] J. Marino, A. Noto and R. Passante, Thermal and Nonthermal Signatures of the Unruh Effect in Casimir-Polder Forces, Phys. Rev. Lett. 113 (2014) 020403 [arXiv:1403.2437] [INSPIRE].

[17] T. Yu and J.H. Eberly, Finite-Time Disentanglement Via Spontaneous Emission, Phys. Rev. Lett. 93 (2004) 140404.

[18] T. Yu and J.H. Eberly, Sudden Death of Entanglement, Science 323 (2009) 598.

[19] Z. Ficek and R. Tanaś, Dark periods and revivals of entanglement in a two-qubit system, Phys. Rev. A 74 (2006) 024304.

[20] D. Braun, Creation of Entanglement by Interaction with a Common Heat Bath, Phys. Rev. Lett. 89 (2002) 277901 [quant-ph/0205019] [INSPIRE].

[21] M.S. Kim, J. Lee, D. Ahn and P.L. Knight, Entanglement induced by a single-mode heat environment, Phys. Rev. A 65, 040101(R) (2002).

[22] S. Schneider and G.J. Milburn, Entanglement in the steady state of a collective-angular-momentum (Dicke) model, Phys. Rev. A 65 (2002) 042107.

[23] A.M. Basharov, Decoherence and entanglement in radiative decay of a diatomic system, J. Exp. Theor. Phys. 94 (2002) 1070.

[24] L. Jakóbczyk, Entangling two qubits by dissipation, J. Phys. A 35 (2002) 6383.

[25] B. Reznik, Entanglement from the vacuum, Found. Phys. 33 (2003) 167 [quant-ph/0212044] [INSPIRE].

[26] F. Benatti, R. Floreanini and M. Piani, Environment Induced Entanglement in Markovian Dissipative Dynamics, Phys. Rev. Lett. 91 (2003) 070402.

[27] Z. Ficek and R. Tanaś, Entanglement induced by spontaneous emission in spatially extended two-atom system, J. Mod. Op. 50 (2003) 2765.

[28] R. Tanaś and Z. Ficek, Entangling two atoms via spontaneous emission, J. Opt. B 6 (2004) S90.

[29] R. Tanaś and Z. Ficek, Sudden birth and death of entanglement of two atoms in a finite temperature reservoir, Phys. Scripta T 140 (2010) 014037.

[30] Z. Ficek and R. Tanaś, Delayed sudden birth of entanglement, Phys. Rev. A 77 (2008) 054301. 
[31] L. Mazzola, S. Maniscalco, J. Piilo, K.-A. Suominen and B.M. Garraway, Sudden death and sudden birth of entanglement in common structured reservoirs, Phys. Rev. A 79 (2009) 042302 .

[32] S. Das and G.S. Agarwal, Bright and dark periods in the entanglement dynamics of interacting qubits in contact with the environment, J. Phys. B 42 (2009) 141003.

[33] F. Benatti and R. Floreanini, Entanglement generation in uniformly accelerating atoms: Reexamination of the Unruh effect, Phys. Rev. A 70 (2004) 012112.

[34] J. Hu and $\mathrm{H} . \mathrm{Yu}$, Entanglement dynamics for uniformly accelerated two-level atoms, Phys. Rev. A 91 (2015) 012327 [arXiv: 1501.03321] [INSPIRE].

[35] Y. Yang, J. Hu and H. Yu, Entanglement dynamics for uniformly accelerated two-level atoms coupled with electromagnetic vacuum fluctuations, Phys. Rev. A 94 (2016) 032337 [arXiv: 1609.06820] [INSPIRE].

[36] S. Cheng, H. Yu and J. Hu, Entanglement dynamics for uniformly accelerated two-level atoms in the presence of a reflecting boundary, Phys. Rev. D 98 (2018) 025001 [arXiv: 1806.05344] [INSPIRE].

[37] J. Zhang and H.W. Yu, The Unruh effect and entanglement generation for accelerated atoms near a reflecting boundary, Phys. Rev. D 75 (2007) 104014 [arXiv: 0705.1092] [InSPIRE].

[38] Y. Zhou, H. Yu and Z. Zhu, Spontaneous Excitation of an Accelerated Detector Interacting with a Massive Scalar Field and Unruh Effect, Commun. Theor. Phys. 57 (2012) 387.

[39] W.G. Brenna, R.B. Mann and E. Martín-Martínez, Anti-Unruh Phenomena, Phys. Lett. B 757 (2016) 307 [arXiv: 1504.02468] [INSPIRE].

[40] L.J. Garay, E. Martín-Martínez and J. de Ramón, Thermalization of particle detectors: The Unruh effect and its reverse, Phys. Rev. D 94 (2016) 104048 [arXiv:1607. 05287] [INSPIRE].

[41] Y. Zhou, J. Hu and H. Yu, Entanglement dynamics for two-level quantum systems coupled with massive scalar fields, Phys. Lett. A 406 (2021) 127460.

[42] V. Gorini, A. Kossakowski and E.C.G. Sudarshan, Completely Positive Dynamical Semigroups of N Level Systems, J. Math. Phys. 17 (1976) 821 [InSPIRE].

[43] G. Lindblad, On the Generators of Quantum Dynamical Semigroups, Commun. Math. Phys. 48 (1976) 119 [INSPIRE].

[44] R. Kubo, Statistical-Mechanical Theory of Irreversible Processes. I. General Theory and Simple Applications to Magnetic and Conduction Problems, J. Phys. Soc. Jpn. 12 (1957) 570.

[45] P.C. Martin and J.S. Schwinger, Theory of many particle systems. 1, Phys. Rev. 115 (1959) 1342 [INSPIRE].

[46] R. Haag, N.M. Hugenholtz and M. Winnink, On the Equilibrium states in quantum statistical mechanics, Commun. Math. Phys. 5 (1967) 215 [inSPIRE].

[47] R. Tanaś, Evolution of quantum correlations in a two-atom system, Phys. Scripta T 153 (2013) 014059.

[48] W.K. Wootters, Entanglement of formation of an arbitrary state of two qubits, Phys. Rev. Lett. 80 (1998) 2245 [quant-ph/9709029] [INSPIRE].

[49] A.P.C.M. Lima, G. Alencar and R.R. Landim, Asymptotic states of accelerated qubits in nonzero background temperature, Phys. Rev. D 101 (2020) 125008 [arXiv:2002.02020] [INSPIRE]. 
[50] G. Kaplanek and C.P. Burgess, Hot Accelerated Qubits: Decoherence, Thermalization, Secular Growth and Reliable Late-time Predictions, JHEP 03 (2020) 008 [arXiv: 1912.12951] [INSPIRE].

[51] T. Li, B. Zhang and L. You, Would quantum entanglement be increased by anti-Unruh effect?, Phys. Rev. D 97 (2018) 045005 [arXiv: 1802.07886] [InSPIRE].

[52] S. Barman and B.R. Majhi, Radiative process of two entangled uniformly accelerated atoms in a thermal bath: a possible case of anti-Unruh event, JHEP 03 (2021) 245 [arXiv:2101.08186] [INSPIRE]. 